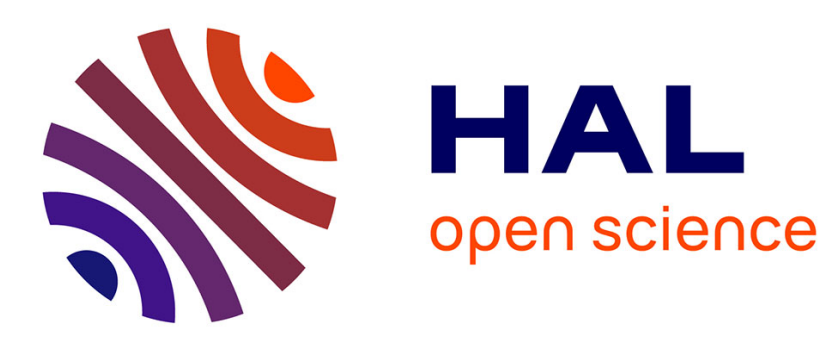

\title{
Distinct pathogenic mechanisms of various RARS1 mutations in Pelizaeus-Merzbacher-like disease
}

Guang Li, Gilbert Eriani, En-Duo Wang, Xiao-Long Zhou

\section{To cite this version:}

Guang Li, Gilbert Eriani, En-Duo Wang, Xiao-Long Zhou. Distinct pathogenic mechanisms of various RARS1 mutations in Pelizaeus-Merzbacher-like disease. Science China Life Sciences, 2021, 10.1007/s11427-020-1838-2 . hal-03135426

\section{HAL Id: hal-03135426 \\ https://hal.science/hal-03135426}

Submitted on 17 Feb 2021

HAL is a multi-disciplinary open access archive for the deposit and dissemination of scientific research documents, whether they are published or not. The documents may come from teaching and research institutions in France or abroad, or from public or private research centers.
L'archive ouverte pluridisciplinaire HAL, est destinée au dépôt et à la diffusion de documents scientifiques de niveau recherche, publiés ou non, émanant des établissements d'enseignement et de recherche français ou étrangers, des laboratoires publics ou privés. 


\title{
Distinct pathogenic mechanisms of various $R A R S 1$ mutations in Pelizaeus-Merzbacher-like disease
}

\begin{abstract}
Guang $\mathrm{Li}^{1}$, Gilbert Eriani ${ }^{2}$, En-Duo Wang ${ }^{1,3,}{ }^{*}$, Xiao-Long Zhou ${ }^{1, *}$
${ }^{1}$ State Key Laboratory of Molecular Biology, CAS Center for Excellence in Molecular Cell Science, Shanghai Institute of Biochemistry and Cell Biology, Chinese Academy of Sciences; University of Chinese Academy of Sciences, 320 Yue-Yang Road, Shanghai 200031; ${ }^{2}$ Architecture et Réactivité de l'ARN, UPR9002 CNRS, Institut de Biologie Moléculaire et Cellulaire, Université de Strasbourg, 15 rue René Descartes, 67084 Strasbourg, France; ${ }^{3}$ School of Life Science and Technology, ShanghaiTech University, Shanghai 201210, China
\end{abstract}

${ }^{*}$ To whom correspondence should be addressed: Prof. En-Duo Wang, Tel.: 86-21-5492-1241; Fax: 86-21-5492-1011; E-mail: edwang@sibcb.ac.cn; Prof. Xiao-Long Zhou, Tel.: 86-215492-1247; Fax: 86-21-5492-1011; E-mail: xlzhou@sibcb.ac.cn; 


\begin{abstract}
Mutations of the genes encoding aminoacyl-tRNA synthetases are highly associated with various central nervous system disorders. Recurrent mutations, including c.[5A>G], p.[D2G]; c.[1367C > T], p.[S456L]; c.[1535G>A], p.[R512Q] and c.[1846_1847del], p.[Y616Lfs*6] of RARS1 gene, which encodes two forms of human cytoplasmic arginyl-tRNA synthetase (hArgRS), are linked to Pelizaeus-Merzbacher-like disease (PMLD) with unclear pathogenesis. Among these mutations, c. $[5 \mathrm{~A}>\mathrm{G}]$ is the most extensively reported mutation, leading to a p.[D2G] mutation in the N-terminal extension of the long-form hArgRS. Here, we showed the detrimental effects of R512Q substitution and $\Delta \mathrm{C}$ mutations on the structure and function of hArgRS, while the most frequent mutation c.[5A>G], p.[D2G] acted in a different manner without impairing hArgRS activity. The nucleotide substitution c. $[5 \mathrm{~A}>\mathrm{G}]$ reduced translation of hArgRS mRNA, and an upstream open reading frame contributed to the suppressed translation of the downstream main ORF. Taken together, our results elucidated distinct pathogenic mechanisms of various RARS1 mutations in PMLD.
\end{abstract}

\title{
KEYWORDS:
}

aminoacyl-tRNA synthetase (aaRS), central nervous system (CNS), protein biosynthesis, translation initiation, tRNA

\section{INTRODUCTION}

Aminoacyl-tRNA synthetases (aaRSs) play critical roles in protein biosynthesis by catalyzing aminoacylation of tRNAs with cognate amino acids to form aminoacyl-tRNAs (aatRNAs) to supply materials for protein biosynthesis. This process usually takes place as a twostep reaction, where the cognate amino acid is first activated by ATP to form aminoacyl-AMP and pyrophosphate, and the aminoacyl moiety is then transferred to the 2'- or 3'-hydroxyl group on the ribose ring of tRNA CCA end to form aa-tRNA. Twenty aaRSs corresponding to cognate amino acids are partitioned into two classes according to the structural motifs in the active site, with 10 aaRSs in each class (Eriani et al., 1990). Class I aaRSs have two consensus signature peptides "HIGH" and "KMSK" in Rossmann fold domain in active sites, and class II aaRSs share three homologous motifs (Eriani et al., 1990). Most aaRSs activate cognate amino acids in the absence of tRNA, with the exception of arginyl-tRNA synthetase (ArgRS, EC 6.1.1.19), glutamyl-tRNA synthetase (GluRS, EC 6.1.1.17) and glutaminyl-tRNA synthetase (GlnRS, EC 6.1.1.18), which activate cognate amino acids in a tRNA-dependent manner (Mehler et al., 1967; Papas et al., 1972). To avoid the mis-incorporation of non-cognate amino acids or other amino acid analogs during protein biosynthesis, aaRSs employ double-sieve mechanism to clear the mis-activated amino acids or mis-charged tRNAs (Nureki et al., 1998). Some aaRSs utilize pretransfer and/or post-transfer editing activity to hydrolyze the mis-activated or mis-charged 
products. In specific cases, the mis-charged are cleared by non-aaRS trans-acting factors (Zhou et al., 2013).

It is typically believed that human genome encodes 37 aaRSs, with 18 aaRSs functioning exclusively in the cytoplasm and 17 functioning exclusively in mitochondria (Bonnefond et al., 2005), while KARSI (encoding lysyl-tRNA synthetase, LysRS) and GARS1 (encoding glycyltRNA synthetase, GlyRS) encode cognate dual-localized aaRSs functioning in both the cytoplasm and mitochondria through alternative mRNA splicing and translation initiation, respectively (Mudge et al., 1998; Shiba et al., 1994; Tolkunova et al., 2000; Wang et al., 2020; Zhou et al., 2017). An additional gene resulting from the duplication of TARS1 gene (encoding cytoplasmic threonyl-tRNA synthetase, ThrRS), named TARS3, has recently been reported to encode ThrRS-like protein (ThrRS-L) that is active in aminoacylation and editing in vitro (Chen et al., 2018; Zhou et al., 2013; Zhou et al., 2019).

AaRSs from eukaryotes have acquired novel structures compared with their prokaryotic counterparts, characterized by inserted or extended domains and the multiple synthetase complex (MSC). The novel structures in aaRSs not only regulate their enzymatic activities, but are also involved in their noncanonical functions. In human cells, the MSC is composed of 9 cytosolic aaRSs and 3 non-enzymatic aaRS-interacting multifunctional proteins (AIMP1, 2 and 3) (Guo et al., 2014). In addition, ThrRS-L is also a component of cytosolic MSC (Zhou et al., 2019). The critical role of aaRSs in protein biosynthesis makes the organism susceptible to any mutations that alter either their catalytic or noncanonical functions. Mutations of aaRS genes are correlated with a diversity of inherited disorders, and the nervous system is the most frequently affected (Gonzalez-Serrano et al., 2019). The widely studied peripheral neuropathy Charcot-Marie-Tooth disease (CMT) is associated with gene mutations of 6 aaRS members (Wei et al., 2019). Furthermore, mutations of both cytoplasmic and mitochondrial aaRS genes are correlated with a wide range of central nervous system (CNS) pathologies, including axonal neuropathy, leukoencephalopathy and demyelination (Jordanova et al., 2006; Latour et al., 2010; Scheper et al., 2007; Steenweg et al., 2012; Taft et al., 2013). In some cases, mutations of aaRS are associated with even more severe syndromes, such as early-onset pontocerebellar hypoplasia type 6 (PCH6), early-onset epileptic encephalopathies (EOEE) and Leigh's syndrome (Edvardson et al., 2007; Kodera et al., 2015; Schwartzentruber et al., 2014; Simon et al., 2015).

ArgRS belongs to class I aaRS. According to the structure of Saccharomyces cerevisiae cytoplasmic ArgRS (ScArgRS), ArgRS contains five functional domains: N-terminal addition domain (Add1) responsible for interacting with D-loop of tRNA ${ }^{\text {Arg; }}$; the catalytic domain; two domains (Ins1 and Ins2) inserted in catalytic domain responsible for closing and connecting two halves of Rossmann fold; and a conserved C-terminal domain responsible for binding and recognizing anticodon loop (Cavarelli et al., 1998). ArgRSs from higher eukaryotes have 
acquired appended N-terminal domain (NTD). Based on the architecture of active site, ArgRS could be classified into three subsets. The first subset, which contains ArgRSs from most species, lacks the canonical "KMSK" signature peptide. In this subset of ArgRSs, a conserved lysine residue two residues upstream of "HIGH" sequence (HIGH-loop lysine) spatially resembles the second lysine in missing "KMSK" to interact with $\alpha$-phosphate to stabilize the transition state of adenylate in Arg activation step (Sekine et al., 2001) (Figure S1A), hence this class of ArgRSs is named "HIGH-loop lysine" group. Several ArgRSs from this subset contain "KMSK"-like sites, like "KFKT" or "PFKT" peptides, but the position of the second lysine critical for Arg activation is shifted compared with the classic "KMSK" signature motif (Figure S1B). The second subset of ArgRSs is named "KMSK group", as they preserve the classic "KMSK" peptide, and a conserved glycine two residues upstream of "HIGH" motif has mutual compensation for Arg activation activity with the "KMSK" signature peptide (Li et al., 2003) (Figure S1B). The last subset contains ArgRSs from several archaea, which lack both the "KMSK" motif and "HIGH-loop" lysine residue; hence they are called the "tentative group" as they might have different catalytic mechanism and/or active site architecture from other class I aaRSs (Sekine et al., 2001) (Figure S1B).

Cytoplasmic ArgRS (encoded by RARS1 in human) is a component of the MSC. Two forms of ArgRS exist in mammalian cells, and ArgRS within the complex has an additional N-terminal domain (NTD) compared with its free-form counterpart (Sivaram et al., 1990; Vellekamp et al., 1987). The NTD of human cytosolic ArgRS (abbreviated as hArgRS in this work) is composed of 72-amino acid residues (Sivaram et al., 1990). It was supposed that the two forms of hArgRS have distinct functions: the complexed long-form hArgRS supplies Arg-tRNA ${ }^{\text {Arg }}$ for protein biosynthesis, while the free short-form enzyme provides Arg-tRNA ${ }^{A r g}$ for protein arginyltransferase (encoded by ATE1 in human) involved in N-terminal arginylation modification of certain protein targeted for ubiquitin-dependent degradation (Ferber et al., 1987; Sivaram et al., 1990). In human cells, the presence of two forms of hArgRS in cells is an indication of the MSC channeling function during protein biosynthesis (Kyriacou et al., 2008; Negrutskii et al., 1991). Our lab mapped the 5'-end of hArgRS mRNA and revealed that the two forms of hArgRS are produced from two alternative translation initiation sites (TIS), AUG1 and AUG2, of a single mRNA transcript, while an upstream AUG start codon (uAUG) initiates an upstream open reading frame (uORF) (Figure 1A). This overlapping uORF initiates at the 8 site and terminates at the +67 site upstream of AUG2, containing 24 sense codons (Figure 1A) (Zheng et al., 2006).

Pelizaeus-Merzbacher disease (PMD, OMIM 312080) is characterized by early-onset hypomyelinating leukodystrophy type 1 (HLD1) typically caused by mutations or rearrangements of the PLP1 gene (OMIM 300401), which encodes two splice variants of a proteolipid in the CNS (Brender et al., 2015; Lossos et al., 2015). A group of disorders in the 
CNS with similar phenotypes (classified as PMD-like disease, PMLD) were reported to be linked to mutations of the genes DARSI (OMIM 603084), RARSI (OMIM 107820) and AIMPI (OMIM 603605) encoding aspartyl-tRNA synthetase (AspRS), arginyl-tRNA synthetase (ArgRS) and non-enzymatic protein AIMP1 of the MSC, respectively (Feinstein et al., 2010; Mendes et al., 2020; Nafisinia et al., 2017; Taft et al., 2013; Wolf et al., 2014). Among these reported mutations, RARS1 mutations are the most frequently identified in patients presenting hypomyelination. Recently, the spectrum of hypomyelination associated with RARS1 mutations was classified based on the clinical presentations of 20 patients, and the mutations identified in more than one family were classified as recurrent mutations (Mendes et al., 2020; Nafisinia et al., 2017; Wolf et al., 2014). However, the genotype-phenotype relationship in RARSIassociated PMLD patients remains elusive.

In this study, we investigated the genetic and molecular mechanisms underlying PMLD caused by recurrent RARS1 mutations. We reveal that conserved substitution or truncation in the Add2 domain of hArgRS decreases enzymatic activity and causes protein degradation in cells, indicating loss-of-function alleles. Specifically, we investigated the most recurrent c. $[5 \mathrm{~A}>\mathrm{G}]$ substitution and found that this substitution does not interfere with enzyme structure or aminoacylation activity but results in a significantly decreased amount of the long-form hArgRS in cells through altered translation initiation.

\section{RESULTS}

\section{Recurrent pathogenic mutations of RARS1 cause mutations in the NTD and/or Add2 domain of hArgRS.}

The PMLD-associated mutations of RARS1 were first separately identified by whole exome sequencing (WES) or WES with a filter for HLD1 genes, either with or without clinical research (Nafisinia et al., 2017; Rezaei et al., 2019; Wolf et al., 2014). These mutations induce singlesite substitutions, deletions, mRNA splicing errors or loss of the long-form hArgRS. Subsequently, a study including 20 PMLD patients with RARS1 mutations identified recurrent mutations in unrelated families (Mendes et al., 2020). In total, 25 cases of PMLD were associated with RARS1 mutations. Based on analysis of these mutations, we only selected the recurrent missense mutations present in patients from more than one family including c. [5A>G], p.[D2G]; c.[1367C > T], p.[S456L]; c.[1535G>A], p.[R512Q] (Table 1). In addition, a de novo mutant c.[1846_1847del], p.[Y616Lfs*6] was also included in this study, although it was present in only one patient (Table 1) (Wolf et al., 2014). This mutation induced frame-shift and early termination, thus encoding $\mathrm{hArgRS}-\Delta \mathrm{C}$ with a significant part of Add 2 domain truncated (Table 1).

The most widely distributed c. $[5 \mathrm{~A}>\mathrm{G}]$ mutation proximal to the 5 '-end of mRNA was identified in 10 PMLD patients out of all 25 reported cases. This nucleotide substitution 
c. $[5 \mathrm{~A}>\mathrm{G}]$ is located in both the main ORF and uORF of hArgRS mRNA (Figure 1A). The resultant D2G amino acid substitution is located in Ha helix (composing M1-K30) of NTD according to the hArgRS structure in a MSC sub-complex (PDB 4R3Z, Figure 1B).

The other two single-site substitutions, S456 and R512, are both located near or in the Cterminal Add2 domain of hArgRS. The semi-conserved S456 located in the junction between

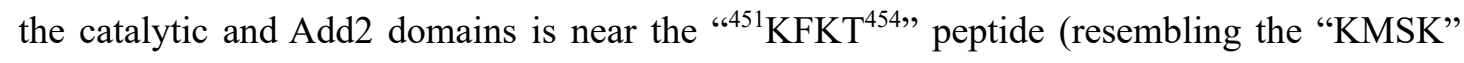
sequence of other class I aaRSs) in primary sequence, while the highly conserved R512 is located in the Add2 domain (Figure 1B and 1C). However, R512 is spatially proximal to catalytic active site (Figure 1B). The last mutation, hArgRS- $\Delta$ C, was created by a 2-nt deletion that induces frame-shift following C615 and early termination at a rebuilt UAG stop codon after adding 5 out-of-frame amino acid residues (-LCGER-) (Figure 1D).

\section{Effects of mutations on the structure and stability of hArgRS in vitro and in vivo.}

Long-form hArgRS and its single-site substitution mutants produced from E. coli transformants containing the related genes were soluble and could be purified to homogeneity, while the truncated mutant $h A \operatorname{rgRS}-\Delta \mathrm{C}$ was insoluble and could not be purified, suggesting instability of the deletion mutant. Although all the single-site mutants were soluble (Figure S2A), substitution of the conserved R512 to Q changed the secondary structures of the enzyme, as shown by shifted absorption at 208-nm and 222-nm of polarized ultraviolet light on its circular dichroism (CD) spectrum, indicating altered secondary structures (Table 2 and Figure S2B). To determine whether the changed secondary structures induced partial misfolding and caused instability of hArgRS mutants, the thermal stabilities were measured by thermal shift assay (TSA), in which a fluorescence dye binds to the hydrophobic regions of the protein during a process of progressive thermal denaturation. Consistent with the results of the CD spectrum, the apparent melting temperatures $\left(\mathrm{T}_{\mathrm{m}}\right)$ of $\mathrm{hArgRS}-\mathrm{D} 2 \mathrm{G}$ and $\mathrm{hArgRS}-\mathrm{S} 456 \mathrm{~L}$ were similar to that of hArgRS, while the $\mathrm{T}_{\mathrm{m}}$ of hArgRS-R512Q increased by approximately $2{ }^{\circ} \mathrm{C}$ (Table 2 and Figure S2C), suggesting that this mutant had a more rigid structure than the wild-type enzyme. These results indicated the importance of the conserved R512 and C-terminal peptide in the Add2 domain of hArgRS for the maintenance of the correct conformation of the enzyme.

To test whether the changed secondary structures and stabilities in vitro occurred in vivo, RARS1 and its mutants were overexpressed in HEK293T cells and compared with the native gene. Overexpression of the RARS1 gene produced both the long-form and short-form hArgRS, and the ratio of long-form hArgRS to short-form hArgRS was approximately 2.5 (Figure 2A), similar to the ratio of native proteins in HEK293T cells $(\sim 3.0)$ reported by our lab (Zheng et al., 2006). However, the expression of different mutant genes yielded distinct amounts of hArgRS variants. First, the two forms of hArgRS-D2G had comparable amounts (the ratio of the longform to the short-form hArgRS was approximately 1.5) because of decreased production of the long-form hArgRS-D2G in vivo, while the other mutants predominantly generated the long- 
form hArgRS (Figure 2A). Furthermore, the amounts of hArgRS-R512Q and hArgRS- $\Delta$ C, either the long-form or short-form protein, were decreased. Only hArgRS-S456L produced similar amounts of the two forms of the protein compared with hArgRS (Figure 2A). A simple explanation for the decreased amount of hArgRS mutants is that it results from the intrinsic instability of these mutants due to changed conformations; hence, the decay rates of the diseasecausing mutants in cells were assayed by cycloheximide ( $\mathrm{CHX}$ ) decay assay. The results showed that hArgRS-D2G and hArgRS-S456L were rather stable with a moderate decrease 12 $\mathrm{h}$ after treatment; however, the amounts of hArgRS-R512Q and hArgRS- $\Delta \mathrm{C}$ were reduced to $50 \%$ of the initial amounts $6 \mathrm{~h}$ after treatment (Figure 2B and Figure 2C), indicating decreased stabilities of hArgRS-R512Q and hArgRS- $\Delta \mathrm{C}$ in cells.

As the long-form hArgRS is one of the MSC components interacting with AIMP1 via the NTD (Fu et al., 2014), we determined whether the mutations could affect its incorporation into the MSC. Co-immunoprecipitation (Co-IP) in HEK293T cells overexpressing RARS1 or its mutant genes showed that two proteins of the MSC, AIMP1 and GluProRS, were equally pulled down by hArgRS or its single-site substitution mutants, suggesting that these single-site mutants can still incorporate into the MSC; however, hArgRS- $\Delta$ C pulled down less AIMP1 and GluProRS, indicating that the C-terminal truncation impairs the interaction between $\mathrm{hArgRS}$ and MSC components (Figure 2D). Because MetRS and ArgRS are strongly dependent on MSC formation for their stability (Han et al., 2006), the impaired interaction with MSC components may account for the poor stability and rapid degradation of hArgRS- $\Delta \mathrm{C}$ in cells.

\section{Enzymatic activities of hArgRS mutants in vitro and in vivo.}

To study the catalytic functions of hArgRS and its mutants, we assayed the Arg activation and tRNA ${ }^{\text {Arg }}$ aminoacylation activities. The Arg activation and aminoacylation activities of hArgRS-D2G are similar to wild-type enzyme, and hArgRS-S456L activated Arg at similar rates as hArgRS while had a decreased aminoacylation activity (Figure 3A and Table 3). However, the activities of hArgRS-R512Q were abolished (Figure 3A). However, mutation of conserved R512 to Q only slightly affected its binding with tRNA ${ }^{\mathrm{Arg}}$, as indicated by a 1.7-fold increase in the dissociation constant $\left(K_{\mathrm{d}}\right)$ compared with that of the wild-type enzyme (Figure S3A). Furthermore, substitution of conserved R512 with other amino acid residues, including hArgRS-R512K, hArgRS-R512A and hArgRS-R512E, severely impaired its aminoacylation activities (Figure S3B). These results indicate that R512 is crucial for enzyme activities, although substitution of this site only has little effect on tRNA ${ }^{\text {Arg }}$ binding.

To further study the pathogenic effects of RARS1 mutations in vivo, we performed complementation assay in a yeast strain with knock-out of $Y D R 341 C$ gene (encoding $S c$ ArgRS) (Figure S4A), and this strain is named $S c \triangle Y D R 341 C$. However, despite efficient expression in yeast cells, hArgRS failed to rescue $S c \triangle Y D R 341 C$ (Figure S4B), as hArgRS was unable to charge one of the four SctRNA ${ }^{\mathrm{Arg}}$ isoacceptors with a -CCG anticodon (Figure S4C). We 
further constructed a hybrid gene encoding chimeric NTD-ScArgRS in which the first 30 amino acid residues (M1 to N30) of ScArgRS were replaced by the NTD (M1 to N72) of hArgRS, and this hybrid gene was able to support the growth of $S c \triangle Y D R 341 C$ (Figure 3B). Furthermore, the mutation sites corresponding to human hArgRS were separately introduced into the chimeric enzyme as following: D2G in the appended NTD, R467Q of NTD-ScArgRS homologous to R512Q of hArgRS in the Add2 domain and NTD-ScArgRS- $\Delta$ C40 to mimic the hArgRS- $\Delta \mathrm{C}$, respectively (Figure 3B, left panel). Among these mutants, NTD-ScArgRS variants containing the single-site substitutions were able to support the growth of Sc $\triangle Y D R 341 C$ with similar amounts of recombinant proteins in yeast cells (Figure 3B, right panel and Figure S4D), while NTD-ScArgRS- $\Delta$ C40 could not complement the function of $S c$ ArgRS and had a decreased protein production in $S c \triangle Y D R 341 C$ (Figure 3B, right panel and Figure S4D). This result indicates different in vivo effects of these mutations on hArgRS and $S c$ ArgRS. First, D2G in NTD neither inhibits $S c$ ArgRS activity nor affects the production of the chimeric enzyme as indicated by the unaffected growth of $S c \triangle Y D R 341 C$ transformant and protein amount (Figure 3B, right panel and Figure S4D). Second, ScArgRS-R467Q is active both in vivo (Figure 3B, right panel and Figure S4D) and in vitro (Figure S4E). Furthermore, a conserved Add2 domain is indispensable for ArgRS activities, as the truncated NTD$S c$ ArgRS- $\triangle$ C40 failed to rescue $S c \triangle Y D R 341 C$ and its protein amount was decreased (Figure 3B, right panel and Figure S4D).

The different effects of Add2 domain mutations on hArgRS and ScArgRS exemplifies the importance of interdomain communication in ArgRS. For instance, mutation of conserved R512 to Q abolished hArgRS activities, while $S c$ ArgRS-R467Q was active in charging tRNA ${ }^{\text {Arg }}$ both in vivo and in vitro. The divergent outcomes of this substitution could be explained by the different architectures of the active site. While ScArgRS and hArgRS both lack the canonical "KMSK" signature motif in the ATP-binding pocket, hArgRS possesses a resembling

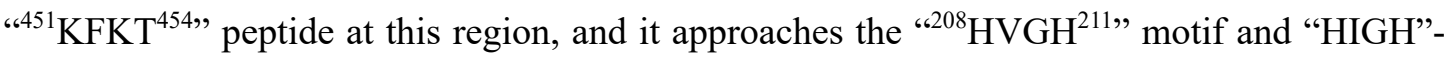
loop K in active site (PDB 4Q2T) (Figure 3C). However, ScArgRS completely lacks a similar sequence and the conformation of this peptide is disordered (PDB 1F7V) (Figure 3C). The different conformations of the active sites might produce different outcomes for ArgRS mutants from different species containing the conserved $\mathrm{R}$ to $\mathrm{Q}$ substitution in Add2 domain, as this site proximal to active site is located in a knot correlating the active site conformation change and tRNA $^{\text {Arg }}$ anticodon recognition (Delagoutte et al., 2000), In addition, truncation of the Cterminal peptide in both hArgRS- $\Delta \mathrm{C}$ and NTD-ScArgRS- $\Delta \mathrm{C} 40$ severely disrupts recognition of tRNA ${ }^{\text {Arg }}$ identity determinants in the anticodon loop, including C35 and A38 of tRNA ${ }^{\text {Arg }}$, mediated by C-terminal peptide (Delagoutte et al., 2000), thus impairing overall protein structures and tRNA ${ }^{\mathrm{Arg}}$-dependent catalytic activities.

ArgRS main ORF translation is decreased by the c. $[5 \mathrm{~A}>\mathrm{G}]$ mutation in the absence of 5'- 


\section{UTR.}

As the above results showed that hArgRS-D2G was not affected in its folding, stability, incorporation into the MSC and catalytic activities, we hypothesized that the decreased production of long-form hArgRS might account for its pathogenicity. Hence, we investigated the transcription and translation process of this mutant. First, the 5'-UTR (G-51 to $\mathrm{G}_{-1}$ in Figure 1A) of hArgRS mRNA was removed to investigate the expression of gene mutants without the side effects of the leader sequence. Furthermore, to compare both mRNA and protein of the mutant gene, the coding sequence of enhanced green fluorescence protein $(E G F P)$ with $\mathrm{C}$ terminal FLAG-tag was fused downstream of hArgRS main ORF (Figure 4A). We determined how different nucleotides in codon 2 affected mRNA and protein production by individually mutating the three nucleotides in codon 2 to test their effects on the expression of the fusion gene. For example, the first $\mathrm{G}_{+4}$ in $\mathrm{G}_{+4} \mathrm{~A}_{+5} \mathrm{C}_{+6}$ was changed to $\mathrm{C}$, $\mathrm{A}$ and $\mathrm{T}$; the second $\mathrm{A}_{+5}$ was changed to $\mathrm{T}, \mathrm{G}$, and $\mathrm{C}$; and the third $\mathrm{C}_{+6}$ was changed to $\mathrm{T}$, $\mathrm{A}$ and $\mathrm{G}$, respectively. qPCR assay of the overexpressed genes using primers specific for $E G F P$ gene showed an unaffected mRNA production from these mutants (Figure 4B).

Despite the mRNA production was not affected by mutation of codon 2, the production of the long-form hArgRS from the above mutants was different. First, the fusion gene produced more short-form protein than the long-form protein, which might result from an altered stability of long-form hArgRS when fused with EGFP; in addition, the molecular mass of the fusion protein hArgRS-EGFP was $\sim 100 \mathrm{kDa}$, larger than that of native hArgRS (Figure 4C). For protein production from the mutant genes, we only investigated the formation of the long-form hArgRS containing D2. The substitution of $\mathrm{G}_{+4}$ to any other nucleotide decreased the formation of the long-form hArgRS-EGFP (Figure 4C), validating the importance of optimal translation initiation context, namely, the Kozak sequence in which eukaryotic ribosomes prefer to start translation on " $\mathrm{R}_{-3} \mathrm{CCA}_{+1} \mathrm{UGG}_{+4}$ " sequences and a purine at -3 and guanine at +4 are the most favorable residues for optimal translation initiation (Kozak, 1981; Kozak, 1984), where the adenine nucleotide of AUG was designated as +1 . For substitution of nucleotide $\mathrm{A}_{+5}$ in codon 2, both $\mathrm{GU}_{+5} \mathrm{C}$ (codon of $\mathrm{V}$ ) and disease-causing $\mathrm{G}_{+4} \mathrm{G}_{+5} \mathrm{C}_{+6}$ (codon of $\mathrm{G}$ ) decreased the longform hArgRS, similar to the effect observed with the $\mathrm{G}_{+4}$ substitution, while $\mathrm{G}_{+4} \mathrm{C}_{+5} \mathrm{C}_{+6}$ (encoding hArgRS-D2A) was capable of initiating translation of the long-form hArgRS, although the protein amount remained low compared to that of native codon 2 (Figure 4C). Combined with the little affected mRNA expression by the above mutations, the results indicate that $\mathrm{A}_{+5}$ of hArgRS mRNA is involved in the translational regulation of long-form hArgRS, similar to the role of the Kozak context in translation initiation. In the substitution mutation of $\mathrm{C}_{+6}$ of codon 2, hArgRS-D2E produced from either $\mathrm{GAA}_{+6}$ or $\mathrm{GAG}_{+6}$ generated similar amount of long-form hArgRS, and the synonymous substitution $\mathrm{GAU}_{+6}$ even produced increased amount of hArgRS (Figure 4C). Collectively, the 3 nucleotides $\left(\mathrm{G}_{+4} \mathrm{~A}_{+5} \mathrm{C}_{+6}\right)$ of codon 2 in 
hArgRS mRNA, especially $\mathrm{G}_{+4}$ and $\mathrm{A}_{+5}$, have been fine-tuned for optimal production of longform $\mathrm{hArgRS}$ during translation, and the disease-causing mutation at $\mathrm{A}_{+5}$ affects the production of the long-form hArgRS by altering translation from the AUG1 start codon without affecting mRNA production.

As the leader sequence of the mRNA transcript plays a role in regulating the production of the two forms of hArgRSs (Zheng et al., 2006), we determined whether the 5'-UTR could differentially affect the production of hArgRS and hArgRS-D2G. The amounts of the long-form hArgRS were not obviously changed with or without the 5'-UTR sequence; however, the 5'UTR efficiently suppressed the production of long-form hArgRS-D2G (Figure 5A), indicating that the 5'-UTR inhibits the production of hArgRS-D2G. Without the 5'-UTR, production of hArgRS-D2G was impaired compared with that of hArgRS but to a less extent than in the presence of the 5'-UTR (1.8-fold versus 19.4-fold in Figure 5A, right panel), indicating that the 5'-UTR promotes the decreased translation of hArgRS mRNA containing the diseasecausing c. $[5 \mathrm{~A}>\mathrm{G}]$ mutation.

\section{uORF inhibits translation of hArgRS mRNA containing c. [5A>G] mutation.}

As mentioned above, a prominent feature of the 5'-UTR is that it contains an overlapping uORF starting from the -8 site $\left(\mathrm{A}_{-8} \mathrm{UG}\right)$ and terminating at the +67 site $\left(\mathrm{U}_{+65} \mathrm{G}_{+66} \mathrm{~A}_{+67}\right)$ upstream of AUG2 (Figure 1A). Our previous studies have shown that this uORF could be readily translated (Zheng et al., 2006). In different contexts, uORFs have been reported to repress or enhance translation of the downstream main ORF, and in some cases, uORFs act as stressdependent regulatory elements (Hinnebusch, 2005; Young et al., 2016). However, the uORF, which is unlikely to be translated with a non-AUG codon (by mutating uAUG to uAUU), is still effective to suppress initiation from AUG1 in mutant mRNA containing c. [5A>G] (Figure 5A, right panel). This indicates that the uORF may regulate translation initiation from AUG1 even it is not translated by introducing a non-uAUG codon.

To directly correlate the translation of the main ORF and uORF, we fused the sequence encoding a partial NTD (M1-L22, named Nt hArgRS) with the F-Luc gene in the presence of uORF. By inserting 2-nt $\left(\mathrm{A}_{+65} \mathrm{U}_{+66}\right)$ at the junction of uORF and $F-L u c$, the overlapping region was extended from 64-nt to 66-nt encoding 22 amino acids in NTD, thus making the DNA fragment encoding Nt hArgRS and $F-L u c$ gene in one open reading frame, while the uORF was out of this frame (Figure 5B). In this construct, translation from AUG1 produced Nt hArgRSluciferase with luciferase activity, while translation from uAUG caused a frameshift and early termination in the F-Luc coding region $\left(\mathrm{U}_{+86} \mathrm{~A}_{+87} \mathrm{~A}+88\right)$ (Figure 5B). Although mRNA production of fusion gene containing c. [5A $>$ G] was not significantly changed (Figure 5C), the resulting protein $\mathrm{Nt}$ hArgRS-D2G-luciferase was decreased compared with that of the native construct, as shown both by the relative activities of the reporter gene (Figure 5D, left panel) and the amount of recombinant protein (Figure 5D, right panel). This result reveals that the 
uORF plays a role in the suppressed translation of the downstream main ORF in c. [5A>G] mutant, thus contributing to the decreased production of the long-form hArgRS-D2G.

\section{DISCUSSION}

\section{Phenotype-genotype relationship in RARS1-related PMLD.}

The severity of typical PMD caused by the PLP1 mutation is classified by the clinical signs (Hobson et al., 2012). Recently, the PMLD spectrum was classified as severe, intermediate, and mild based on the clinical presentations of patients harboring RARS1 mutations (Mendes et al., 2020). The molecular basis for its pathogenicity is expected to reveal genotype-phenotype relationships. In this study, we investigated the recurrent mutations of RARS1 associated with PMLD on molecular level, and revealed a dual-regulatory mechanism with different domains involved: mutations in the C-terminal Add2 domain impaired protein structure and catalytic activities, while substitution in eukaryote-specific NTD affected protein production through translational regulation.

Generally, among the studied recurrent mutations of RARS1 gene, mutations that severely affect hArgRS activity or protein structure are associated with severe symptoms (Mendes et al., 2020), which can be exemplified by the severe phenotype of patients containing Add 2 domain mutations of hArgRS. For instance, both patients carrying c.[1535G>A], p.[R512Q] presented severe phenotypes, including early-onset nystagmus, microcephaly, hypomyelination and brain atrophy (Mendes et al., 2020), and it should be noted that c.[1535G $>A$ ], p.[R512Q] substitution is complex heterozygous with AUG1 mutations c.[1A>G]; [1535G>A], p.[0?]; [R512Q] in 1 patient and c.[2T $>$ C]; [1535G $>A$ ], p.[0?]; [R512Q]. Although the expression of AUG1 mutants was not assayed in this study, the abolished production of the long-form hArgRS due to AUG1 mutations is expected to have a detrimental effect on protein biosynthesis in the CNS when presented in the homozygous form or complexed with a function-null allele such as c.[1535G $>$ A], p.[R512Q]. For other mutations in the Add2 domain of hArgRS, the genotypephenotype relationship presented a similar pattern. For instance, the phenotypes associated with the c.[1367C $>\mathrm{T}], \mathrm{p}[\mathrm{S} 456 \mathrm{~L}]$ substitution varied in different patients: a patient carrying c.[1367C >T]; [1846_1847del], p.[S456L]; [Y616Lfs*6] complex heterozygous mutation presented a severe phenotype as hArgRS-S456L had a compromised activity and hArgRS- $\Delta \mathrm{C}$ was inactive and unstable, while c.[1367C $>$ T]; [475C $>$ T], p.[S456L]; [P159S] induced mild symptoms (Mendes et al., 2020), indicating that the detrimental effects on protein functions caused by mutations in the Add2 domain are associated with severe pathogenic consequences.

Although most frequently related to PMLD, the c. [5A $>\mathrm{G}]$ mutation of $R A R S 1$ only caused mild or intermediate symptoms (Mendes et al., 2020; Nafisinia et al., 2017), suggesting a less affected protein function by this mutation. The mild symptoms of patients carrying homozygous c. $[5 A>G] ;[5 A>G]$ indicate that the decrease in the long-form hArgRS-D2G accounts for its 
pathogenicity, but induces a less severe phenotype possibly due to its intact catalytic activity, MSC incorporation and protein stability. However, the long-form hArgRS is indispensable for protein biosynthesis as revealed by the severe phenotypes of patients carrying the start codon AUG1 mutation either in homozygous form or complex heterozygous form with a functionnull allele (Mendes et al., 2020; Rezaei et al., 2019), while a low level of the long-form hArgRS-D2G alleviates the symptom, as observed in a patient carrying c.[5A>G]; [96_97del], p.[D2G]; [C32Wfs*39] complex heterozygous mutation (Mendes et al., 2020; Wolf et al., 2014). As the long-form ArgRS is essential for protein biosynthesis and cell growth even when the short-form ArgRS is present and has a similar activity in synthesizing ArgRS-tRNA ${ }^{\operatorname{Arg}}$ (Kyriacou et al., 2008), the diminished production of complex-associated hArgRS is sufficient to disrupt protein biosynthesis in CNS but induces a less severe phenotype.

\section{Cis-acting regulation of uORF on hArgRS translation.}

In the directional scanning process of eukaryotic translation initiation, the $43 \mathrm{~S}$ pre-initiation complex (PIC) composed of the ribosomal 40S subunit and initiation factors, including eIF1, eIF1A and eIF2-GTP-Met-tRNA ${ }_{i}{ }^{\text {Met }}$ ternary complex, scans for an optimal start codon in the 5'-3' direction (Hinnebusch et al., 2016; Jackson et al., 2010). Hence, the translation of the main ORF is regulated by both the availability of PIC complex and mRNA secondary structures. uORFs located in the 5'-UTRs serve as important regulators of stress-responsive translational control in yeast and in mammalian cells (Hinnebusch, 2005; Vattem et al., 2004). Generally, translation of the UORF attenuates translation of the downstream main ORF because reinitiation after the uORF is often inefficient. In the specific case of RARS1 gene, the uORF overlaps with the coding region of hArgRS mRNA and translation of the uORF causes the bypass of the AUG1 start codon and generates the short-form hArgRS (Zheng et al., 2006), which may function in $\mathrm{N}$-terminal protein modification and/or ubiquitin-dependent degradation by the N-end rule (Ferber et al., 1987; Sivaram et al., 1990; Varshavsky, 2011).

The present study highlighted the regulatory functions of the overlapping uORF beyond translation. In the PMLD cases associated with RARS1 mutations, the hArgRS-D2G variant does not interfere with protein structure and enzymatic activity. However, the presence of a special 5'-UTR inhibits its translation. Furthermore, we show that the uORF sequence contributes to suppressed translation of the downstream main ORF. c. $[5 \mathrm{~A}>\mathrm{G}]$ is located 10 nucleotides downstream of the $\mathrm{uAUG}$, which means that it has to enter the ribosome mRNA channel before uAUG reaches the P site (approximately 15-nt are covered by the ribosome on both sides of the AUG codon). Although no nucleotide recognition is expected to occur in this part of the mRNA path, the presence of secondary structures at the leading edge of the mRNA path (usually 14-nt after the AUG) may improve translation initiation in poor Kozak contexts (Kozak, 1990).

Here, the pathogenic mutation c. $[5 \mathrm{~A}>\mathrm{G}]$ may affect the local mRNA structure or alter the 
identities of the preferred nucleotide contexts for translation initiation. Generally, TIS selection is efficient when start codon is flanked by conserved $R_{-3}$ ( $R$ for purine nucleotides), and the $G_{+4}$ augments TIS selection when $\mathrm{R}_{-3}$ is replaced by a pyrimidine nucleotide (Kozak, 1986; Kozak, 1987; Kozak, 1997). However, in certain TIS contexts, other nucleotides in coding region including in positions +5 and +6 also affect initiation efficiency, especially for non-AUG TIS selection (Kozak, 1997; Boeck et al., 1994; Grünert et al., 1994). In the specific case of RARS1 selection, translation initiation from AUG1 is prone to leaky-scanning even it is encompassed by optimal Kozak context including $\mathrm{A}_{-3}$ and $\mathrm{G}_{+4}$, and the bypass of $\mathrm{AUG}_{1}$ enables translation re-initiation from downstream AUG2 to produce short-form ArgRS. Nucleotides at +4 and +5 sites are fine-tuned for the equilibrium between leaky-scanning and initiation from AUG1. We further compared the potential secondary structure of the mRNA sequence. The suppressed translation of mutant mRNA containing c.[5A>G] substitution might also result from destabilized hairpin mRNA structure downstream of AUG1 (Figure S5), which might compromise recognition of AUG1 start codon (Kozak, 1990). The impaired translation of c. $[5 \mathrm{~A}>\mathrm{G}]$ mutant implies that hArgRS mRNA developed more sophisticated TIS context to regulate the production of two forms of proteins.

As previously reported, translation of uORF impacts translation of the two forms of hArgRSs (Zheng et al., 2006). Translation of the gene containing c. [5A>G] substitution was suppressed by a special 5'-mRNA structure, and an overlapping uORF was involved in the regulation of altered translation initiation. However, we cannot exclude the regulatory functions of other sequence-based elements in the 5'-UTR. In addition, although the regulated expression of stress-response-related genes mediated by upstream translatable sequences have been studied both in fungal and mammalian cells (Hinnebusch, 2005; Vattem et al., 2004), whether the cisacting translational regulation mediated by the 5 '-leader sequence of the aaRS gene responds to environmental stress or altered nutrient conditions to maintain homeostasis remains to be investigated.

The development of ribosome profiling and sequencing revealed that numerous long noncoding RNAs (IncRNAs) have coding potentials. In a few cases, the peptides containing less that 100 -aa encoded by these lncRNAs are functional in muscle performance and oncogenic signaling suppression (Anderson et al., 2015; Xu et al., 2020). In addition, although a relatively small fraction of up-stream sequences of annotated mRNA coding region encodes peptide with regulatory functions, the short peptide encoded by certain uORFs might cause $80 \mathrm{~S}$ ribosome dissociation or stalling, thus preventing translation of the downstream main ORF (Ivanov et al., 2010; Raney et al., 2002; Wei et al., 2012; Werner et al., 1987). In the case of the uORF of the $R A R S 1$ gene, the regulatory function on translational level of uORF to main homeostasis or MSC function is of great interest. 


\section{MATERIALS AND METHODS}

\section{Materials}

L-Arg, dithiothreitol (DTT), NTP mix, GMP, tetrasodium pyrophosphate, Tris-base, $\mathrm{NaCl}$, $\mathrm{KCl}, \mathrm{MgCl}_{2}$, cycloheximide ( $\mathrm{CHX}$ ) and activated charcoal were purchased from Sigma-Aldrich (St. Louis, MO, USA). L-[2, 3, 4- $\left.{ }^{3} \mathrm{H}\right]$ Arg and tetrasodium $\left[{ }^{32} \mathrm{P}\right]$ pyrophosphate were purchased from PerkinElmer Life Sciences (Waltham, MA, USA). KOD-plus-neo polymerase, KOD-plus mutagenesis kit and SYBR Green realtime PCR master mix were purchased from TOYOBO (Japan). T4 DNA ligase, T4 polynucleotide kinase, restriction endonucleases, inorganic pyrophosphatase, DNase I, TRIzol Reagent, Lipofectamine 2000 transfection reagent, Dynabeads protein $G$ and Protein Thermal Shift dye kit were purchased from Thermo Scientific (Pittsburgh, PA, USA). Minimal SD base, DO supplement -His/-Leu/-Trp/-Ura, Yeastmaker yeast transformation kit and PrimeScript RT reagent kit were purchased from Takara (Japan). Nano-Glo Dual-luciferase reporter assay reagent was purchased from Promega (Madison, WI, USA). $\mathrm{Ni}^{2+}$-NTA Superflow was purchased from Qiagen Inc. (Dusseldorf, Germany). Protease inhibitor cocktail was purchased from Roche (Basel, Switzerland). E. coli Rosetta (DE3) chemically competent cells were purchased from TIANGEN Biotech (Beijing, China). Mouse anti-FLAG mAb (66008-3) and mouse anti-GAPDH mAb (60004-1) were purchased from Proteintech Inc. (Wuhan, China). Rabbit anti-hGluProRS pAb, rabbit anti-AIMP1 pAb and rabbit anti-hArgRS pAb were produced in our lab as reported previously (Lei et al., 2015).

\section{Clinical cases}

The cases with a genetic diagnosis of PMLD were reported previously by other labs (Mendes et al., 2020; Nafisinia et al., 2017; Wolf et al., 2014), and the recurrent variants were identified in a cohort with similar phenotypes (Mendes et al., 2020).

\section{Gene cloning, mutagenesis and protein purification of hArgRS, gene expression and purification of $E c t R N A^{\text {Arg }}$}

The coding sequence of the RARS1 gene (RefSeq NG_041809.1) was amplified from cDNA of HEK293T cells, which was obtained by reverse-transcription PCR of total RNA. Gene mutants were constructed according to the protocol provided by the KOD-Plus mutagenesis kit. For protein purification, the gene encoding hArgRS and its mutants were ligated into pET28a(+) with an N-terminal $\mathrm{His}_{6}$ tag, and the recombinant plasmids were transformed into E. coli Rosetta (DE3) cells. The transformant cells were cultured at $37^{\circ} \mathrm{C}$ till $\mathrm{A}_{600}$ of $0.6 \sim 0.8$, and the expression of the genes was induced by the addition of $150 \mu \mathrm{M}$ isopropyl- $\beta-\mathrm{D}-$ thiogalactopyranoside (IPTG) at $20^{\circ} \mathrm{C}$ for $10 \mathrm{~h}$. Purification of hArgRS and its mutants was performed according to our reported method (Zhou et al., 2008). E. coli tRNA ${ }^{\text {Arg }}$ (EctRNA ${ }^{\text {Arg }}$ ) was prepared by the method reported by our lab (Wu et al., 1999).

\section{Circular dichroism and protein thermal shift assay}

Circular dichroism (CD) absorption spectrum was collected on a Jasco J-715 
spectropolarimeter between wavelengths of 240-nm and 190-nm. Protein solutions were diluted to $0.1 \mathrm{mg} / \mathrm{ml}$ in $10 \mathrm{mM}$ potassium phosphate (pH 7.5). CD signals were recorded at $25^{\circ} \mathrm{C}$ over two accumulative scans after the background signals from the cuvette and buffer were subtracted. The CD signals were converted to molar ellipticity, and the absorption curve was plotted using GraphPad Prism software. The fractions of different secondary structures were calculated by comparing the CD data points between 190-nm and 240-nm at 1-nm interval with the reference spectrum as described previously (Chang et al., 1978).

Thermal shift assay (TSA) was performed on a 7500 Fast Real-Time PCR System (Applied Biosystems) using a Protein Thermal Shift dye kit. The TSA mixture contained $1 \times$ Protein Thermal Shift buffer, $1 \times$ Protein Thermal Shift dye and $0.3 \mathrm{mg} / \mathrm{ml} \mathrm{hArgRS}$ or its variants. Melting curves were recorded during a progressive heating process from $25^{\circ} \mathrm{C}$ to $99{ }^{\circ} \mathrm{C}$ at a $1 \%$ ramp speed. The fluorescent dye ROX was used as a reporter, and a no protein control was used to monitor and subtract the background from each analysis group. The apparent melting temperature $\left(\mathrm{T}_{\mathrm{m}}\right)$ was analyzed with Protein Thermal Shift Software.

\section{ATP-PPi exchange and aminoacylation activity assays}

The arginine activation activities were measured by the ATP-PPi exchange reaction (Fang et al., 2014) in a mixture containing $50 \mathrm{mM}$ Tris- $\mathrm{HCl}$ (pH 7.5), $12 \mathrm{mM} \mathrm{MgCl}_{2}, 80 \mathrm{mM} \mathrm{KCl}, 0.05$ $\mathrm{mg} / \mathrm{ml}$ BSA, 4 mM ATP, 4 mM L-Arg, $10 \mu \mathrm{M}$ EctRNA ${ }^{\mathrm{Arg}}, 2 \mathrm{mM}$ tetrasodium $\left[{ }^{32} \mathrm{P}\right]$ pyrophosphate and $10 \mathrm{nM}$ hArgRS or its variants. The aminoacylation activities were assayed according to our previous method (Zhou et al., 2013) in a reaction mixture containing 50 mM Tris- $\mathrm{HCl}$ (pH 7.5), 12 mM MgCl 2,80 mM KCl, 0.5 mM DTT, 0.05 mg/ml BSA, 4 mM ATP, $5 \mu \mathrm{M}$ htRNA ${ }^{\mathrm{Arg}}, 50 \mu \mathrm{M}\left[{ }^{3} \mathrm{H}\right] \mathrm{Arg}$ and $30 \mathrm{nM}$ ArgRS or its variants. In the assay of the aminoacylation kinetics of hArgRS or its variants for tRNA ${ }^{\mathrm{Arg}}, E c t R N A^{\mathrm{Arg}}$ was used as the substrate because hArgRS can charge EctRNA ${ }^{\text {Arg }}$ (Yang et al., 2010). The concentration of enzyme was set to $5 \mathrm{nM}$ hArgRS or hArgRS-D2G and $10 \mathrm{nM}$ hArgRS-S456L, and the concentrations of $E c t R N A^{\text {Arg }}$ ranged from 0 to $25 \mu \mathrm{M}$. Kinetic constants were calculated with Hyperbolic Regression software v 1.1w (Studnicka, 1987).

\section{Gel shift assay}

tRNA binding of hArgRS or its variant was assayed by gel shift assay. The mixture contains

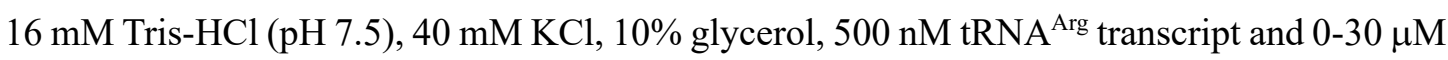
hArgRS or hArgRS-R512Q. The mixture was incubated on ice for $20 \mathrm{~min}$ for specific binding, and the complexed and free forms of tRNA ${ }^{\mathrm{Arg}}$ were separated on a $6 \%$ native polyacrylamide gel by electrophoresis at $4{ }^{\circ} \mathrm{C}$ in $50 \mathrm{mM}$ Tris/glycine buffer. The gel was stained with ethidium bromide to detect tRNA ${ }^{\text {Arg }}$. The amount of free form tRNA ${ }^{\text {Arg }}$ was quantified by analyzing the stained band of tRNA ${ }^{\text {Arg }}$ using MultiGauge software. The amount of tRNA ${ }^{\text {Arg }}$ in the absence of enzyme $(0 \mu \mathrm{M})$ was set to $500 \mu \mathrm{M}$, and the specific binding was quantified by subtracting free form tRNA ${ }^{\text {Arg }}$ from each group. The specific binding between enzyme and tRNA ${ }^{\text {Arg }}$ was fitted 
to a Scatchard curve and dissociation constant $\left(K_{\mathrm{d}}\right)$ was calculated using one-site binding equation.

\section{Construction and complementation of $S c \triangle Y D R 341 C$, preparation of yeast cell lysates and western blot}

The procedure of constructing Sc $Y Y D R 341 C$ is represented in Figure S4A. Briefly, the 5'and 3'-UTR sequences flanking the YDR341C ORF were inserted into the pRS303 vector (containing HIS3 gene), respectively. Digestion of this vector generated a linearized vector in which HIS3 was flanked by the UTRs of YDR341C. This linearized vector was introduced into the BY4741 haploid yeast strain (MATa his $3 \Delta 1$ leu $2 \Delta 0$ met15 $\Delta 0$ ura $3 \Delta 0$ ) containing the rescue plasmid p416TEF-YDR341C, and the YDR341C gene was disrupted by double homologous recombination. The transformants were selected on $\mathrm{SD} / \mathrm{His}^{-}$agar plates and subjected to genotype verification by PCR using primers specific for the 5'- and 3'-UTR sequences.

For complementation assay, the genes encoding NTD-ScArgRS and its variants were inserted into the yeast expression vector p425TEF, and the constructs were introduced into Sc $\triangle Y D R 341 C$. The transformants were selected on $\mathrm{SD} / \mathrm{Ura}^{-} / \mathrm{Leu}^{-}$agar plates and cultured in $\mathrm{SD} / \mathrm{Leu}^{-}$liquid medium. The culture was then diluted to $\mathrm{A}_{600}=1$, and aliquots of 10 -fold serial dilutions of yeast culture were plated on $\mathrm{SD} / \mathrm{Leu}^{-}$agar plates and $\mathrm{SD} / \mathrm{Leu}^{-}$agar plates containing $0.2 \%$ 5-fluoroorotic acid (SD/Leu/5-FOA). The p425TEF empty vector and YDR341C were used as the negative and positive controls, respectively. The culture was incubated at $30^{\circ} \mathrm{C}$, and the growth of the transformants on agar plates was observed.

Transformants selected on SD/Leu agar plates were cultured in SD/Leu- liquid medium, and the yeast cell lysates were prepared according to a previously described method (Jazwinski, 1990). The recombinant proteins in cell lysates were detected by western blot using antihArgRS antibody. GAPDH was used as a standard control. Antibodies used in immunoblotting are diluted in buffer containing $50 \mathrm{mM}$ Tris- $\mathrm{HCl}(\mathrm{pH} 7.5), 150 \mathrm{mM} \mathrm{NaCl}, 0.05 \%$ (v/v) Tween$20,0.03 \mathrm{~g} / \mathrm{ml}$ bovine serum albumin (BSA) and $30 \mathrm{mM}$ sodium azide to a final concentration of $1 \mu \mathrm{g} / \mathrm{ml}$ anti-hArgRS pAb and $1 \mu \mathrm{g} / \mathrm{ml}$ anti-GAPDH mAb.

\section{Cell transfection, co-immunoprecipitation, $\mathrm{CHX}$ treatment and western blot}

The coding sequences with or without 5'-UTR were inserted into pCMV-3T-3A. HEK293T cells were transfected with the construct pCMV-3T-3A-RARSI or its variants to express the genes with a C-terminal $3 \times$ FLAG-tag using Lipofectamine 2000 Reagent according to the manufacturer's instructions. At $24 \mathrm{~h}$ after transfection, cells were harvested and lysed in lysis buffer containing $50 \mathrm{mM}$ Tris- $\mathrm{HCl}$ (pH 7.5), $150 \mathrm{mM} \mathrm{NaCl}, 1 \mathrm{mM}$ EDTA, 1\% NP-40 and 1× protease inhibitor cocktail. After centrifugation at $10,800 \times \mathrm{g}$ at $4{ }^{\circ} \mathrm{C}$, supernatants were collected and subjected to SDS-PAGE and western blot. All antibodies used in immunoblotting were diluted in buffer containing $50 \mathrm{mM}$ Tris- $\mathrm{HCl}$ (pH 7.5), $150 \mathrm{mM} \mathrm{NaCl}, 0.05 \%$ (v/v) Tween$20,0.03 \mathrm{~g} / \mathrm{ml}$ bovine serum albumin (BSA) and $30 \mathrm{mM}$ sodium azide to a final concentration 
of $0.5 \mu \mathrm{g} / \mathrm{ml}$ anti-FLAG mAb, $1 \mu \mathrm{g} / \mathrm{ml}$ anti-AIMP/p43 pAb, $1 \mu \mathrm{g} / \mathrm{ml}$ anti-hGluProRS pAb and $1 \mu \mathrm{g} / \mathrm{ml}$ anti-GAPDH mAb.

For co-immunoprecipitation of MSC components, anti-FLAG mAb was added to the supernatant to a final concentration of $4 \mu \mathrm{g} / \mathrm{ml}$. After incubation for overnight at $4{ }^{\circ} \mathrm{C}$ with gentle agitation, Dynabeads Protein $\mathrm{G}$ was added to the mixture and incubated for $3.5 \mathrm{~h}$ at $4{ }^{\circ} \mathrm{C}$. MSC components were eluted from Protein G beads in $2 \times$ SDS protein loading buffer $(40 \mathrm{mM}$ Tris-HCl, pH 6.8, 1.6\% SDS, $0.2 \%$ bromophenol blue, $20 \%$ glycerol and $80 \mathrm{mM} \mathrm{2-}$ mercaptoethanol) by heating to $95{ }^{\circ} \mathrm{C}$ for $10 \mathrm{~min}$. Cell lysates and immunoprecipitated MSC components were separated by SDS-PAGE and immunoblotted with anti-FLAG, anti-AIMP1 and anti-hGluProRS antibodies, respectively.

For the cycloheximide (CHX) pulse-chase assay, at $24 \mathrm{~h}$ after transfection, $\mathrm{CHX}$ was added to the medium to final concentration $50 \mu \mathrm{g} / \mathrm{ml}$ for indicated time intervals. After CHX treatment, cells were harvested and lysed. The lysates were subjected to western blot and detected by antiFLAG $\mathrm{mAb}$ and anti-GAPDH mAb. The amounts of hArgRS and its mutants after CHX treatment were normalized to the GAPDH amount, as GAPDH is stable in cells after CHX treatment (Gawron et al., 2016). The relative protein amount without CHX treatment $(0 \mathrm{~h})$ was set as $100 \%$ and the amount of the remaining protein was quantified by analyzing western blot bands using MultiGauge software (FUJIFILM).

\section{RNA extraction, reverse-transcription and qPCR analysis of RARS1-EGFP and Nt hArgRS/F-Luc fusion genes}

To analyze the mRNA expression of hArgRS and its pathogenic mutants, related genes were fused to the EGFP gene (encoding enhanced green fluorescence protein) at its 3'-end. The coding sequences of the fusion genes were inserted to pCMV-3T-3A with the start codon AUG of $E G F P$ gene mutated to AUU to block translation initiation from AUG codon of EGFP gene. HEK293T cells were transfected with these constructs to express the fusion genes encoding hArgRS-EGFP or its mutants. At $24 \mathrm{~h}$ after transfection, total RNA was extracted from the harvested cells using TRIzol Reagent. cDNAs were obtained from total RNA using the PrimeScript RT reagent kit and then used for quantitative real-time PCR (qPCR) analysis. qPCR assays were performed using SYBR Green realtime PCR master mix on a LightCycler 96 instrument (Roche) using primers specific for EGFP gene and $A C T B$ gene (encoding beta-actin in human). Expression of $A C T B$ was used for normalization. The relative mRNA levels of each examined gene were calculated using the $2^{-\Delta \Delta \mathrm{C}_{\mathrm{T}}}$ method from the $\mathrm{C}_{\mathrm{T}}$ values obtained from $\mathrm{qPCR}$ assay (Livak et al., 2001).

For analysis of mRNA expression of Nt hArgRS of RARS1 gene, the coding sequences were fused to the firefly luciferase gene $F$-Luc to enable in-frame expression of Nt hArgRS/F-Luc with the start codon of $F-L u c$ gene mutated to AUU, and the fusion gene was inserted into pcDNA3.1. At $24 \mathrm{~h}$ after transfection of HEK293T cells to co-express Nt hArgRS/F-Luc fusion 
genes and the $R$-Luc gene, total RNA was extracted and reverse-transcribed to obtain cDNA. qPCR was performed using primers specific for $F$-Luc gene and $A C T B$ gene, respectively; and expression of $A C T B$ was used for normalization. All the primers used in the qPCR assay are listed in Table S1.

\section{Dual-luciferase assay}

Expression of dual-luciferase reporter genes was used to assay the translation of the coding region of Nt ArgRS or its mutants. At $24 \mathrm{~h}$ after transfection of HEK293T cells to co-express $\mathrm{Nt}$ hArgRS/F-Luc fusion genes and the $R-L u c$ gene as described above, expression of $\mathrm{Nt}$ hArgRS/F-Luc gene or its mutant was quantified by assaying relative luciferase activity using a Dual-Luciferase Reporter Assay kit. Briefly, at $24 \mathrm{~h}$ after co-expressing the $\mathrm{Nt} \mathrm{hArgRS/F-Luc}$ fusion gene and $R$-Luc gene in HEK293T cells, cells were harvested and lysed in Passive Lysis Buffer (PLB). Whole cell lysate was centrifuged at $12,300 \times \mathrm{g}$ at room temperature, and supernatant was collected to detect luciferase activities. Firefly luciferase and Renilla luciferase activities were assayed by recording the cognate bioluminescent signals using a GloMax 20/20 luminometer (Promega) instrument. The relative expression of fusion genes is represented as the ratio of firefly luciferase activity to Renilla luciferase activity.

\section{SUPPLEMENTARY MATERIAL}

Supplementary figure is available for this article.

\section{Compliance and ethics}

The author(s) declare that they have no conflict of interest.

\section{ACKNOWLEDGEMENTS}

This work was supported by the National Key Research and Development Program of China (2017YFA0504000); the Natural Science Foundation of China (91940302, 31500644, 31570792, 31822015, 81870896, 31670801); the Strategic Priority Research Program of the Chinese Academy of Sciences (XDB19010203).

\section{REFERENCES}

Anderson, D. M., Anderson, K. M., Chang, C. L., Makarewich, C. A., Nelson, B. R., McAnally, J. R., Kasaragod, P., Shelton, J. M., Liou, J., Bassel-Duby, R., et al. (2015). A micropeptide encoded by a putative long non-coding RNA regulates muscle performance. Cell 160, 595606.

Bonnefond, L., Fender, A., Rudinger-Thirion, J., Giege, R., Florentz, C. and Sissler, M. (2005). Toward the full set of human mitochondrial aminoacyl-tRNA synthetases: characterization of AspRS and TyrRS. Biochemistry 44, 4805-4816. 
Boeck, R. and Kolakofsky, D. (1994). Positions +5 and +6 can be major determinants of the efficiency of non-AUG initiation codons for protein synthesis. EMBO J 13, 3608-3617.

Brender, T., Wallerstein, D., Sum, J. and Wallerstein, R. (2015). Unusual presentation of Pelizaeus-Merzbacher disease: female patient with deletion of the proteolipid protein 1 gene. Case Rep Genet 2015, 453105.

Cavarelli, J., Delagoutte, B., Eriani, G., Gangloff, J. and Moras, D. (1998). L-rginine recognition by yeast arginyl-tRNA synthetase. EMBO J 17, 5438-5448.

Chang, C. T., Wu, C. S. and Yang, J. T. (1978). Circular dichroic analysis of protein conformation: inclusion of the beta-turns. Anal Biochem 91, 13-31.

Chen, Y., Ruan, Z. R., Wang, Y., Huang, Q., Xue, M. Q., Zhou, X. L. and Wang, E. D. (2018). A threonyl-tRNA synthetase-like protein has tRNA aminoacylation and editing activities. Nucleic Acids Res 46, 3643-3656.

Delagoutte, B., Moras, D. and Cavarelli, J. (2000). tRNA aminoacylation by arginyl-tRNA synthetase: induced conformations during substrates binding. EMBO J 19, 5599-5610.

Edvardson, S., Shaag, A., Kolesnikova, O., Gomori, J. M., Tarassov, I., Einbinder, T., Saada, A. and Elpeleg, O. (2007). Deleterious mutation in the mitochondrial arginyl-transfer RNA synthetase gene is associated with pontocerebellar hypoplasia. Am J Hum Genet 81, 857862.

Eriani, G., Delarue, M., Poch, O., Gangloff, J. and Moras, D. (1990). Partition of tRNA synthetases into two classes based on mutually exclusive sets of sequence motifs. Nature 347, 203-206.

Fang, Z. P., Wang, M., Ruan, Z. R., Tan, M., Liu, R. J., Zhou, M., Zhou, X. L. and Wang, E. D. (2014). Coexistence of bacterial leucyl-tRNA synthetases with archaeal tRNA binding domains that distinguish tRNA ${ }^{\text {Leu }}$ in the archaeal mode. Nucleic Acids Res 42, 5109-5124.

Feinstein, M., Markus, B., Noyman, I., Shalev, H., Flusser, H., Shelef, I., Liani-Leibson, K., Shorer, Z., Cohen, I., Khateeb, S., et al. (2010). Pelizaeus-Merzbacher-like disease caused by AIMP1/p43 homozygous mutation. Am J Hum Genet 87, 820-828.

Ferber, S. and Ciechanover, A. (1987). Role of arginine-tRNA in protein degradation by the ubiquitin pathway. Nature 326, 808-811.

Fu, Y. Y., Kim, Y., Jin, K. S., Kim, H. S., Kim, J. H., Wang, D., Park, M., Jo, C. H., Kwon, N. H., Kim, D., et al. (2014). Structure of the ArgRS-GlnRS-AIMP1 complex and its implications for mammalian translation. Proc Natl Acad Sci U S A 111, 15084-15089.

Gawron, D., Ndah, E., Gevaert, K. and Van Damme, P. (2016). Positional proteomics reveals differences in N-terminal proteoform stability. Mol Syst Biol 12, 858-878.

Gonzalez-Serrano, L. E., Chihade, J. W. and Sissler, M. (2019). When a common biological role does not imply common disease outcomes: disparate pathology linked to human mitochondrial aminoacyl-tRNA synthetases. J Biol Chem 294, 5309-5320. 
Grünert, S. and Jackson, R. J. (1994). The immediate downstream codon strongly influences the efficiency of utilization of eukaryotic translation initiation codons. EMBO J 13, 36183630.

Guo, M. and Yang, X. L. (2014). Architecture and metamorphosis. Top Curr Chem 344, 89-118.

Han, J. M., Lee, M. J., Park, S. G., Lee, S. H., Razin, E., Choi, E. C. and Kim, S. (2006). Hierarchical network between the components of the multi-tRNA synthetase complex: implications for complex formation. J Biol Chem 281, 38663-38667.

Hinnebusch, A. G. (2005). Translational regulation of GCN4 and the general amino acid control of yeast. Annu Rev Microbiol 59, 407-450.

Hinnebusch, A. G., Ivanov, I. P. and Sonenberg, N. (2016). Translational control by 5'untranslated regions of eukaryotic mRNAs. Science 352, 1413-1416.

Hobson, G. M. and Garbern, J. Y. (2012). Pelizaeus-Merzbacher disease, PelizaeusMerzbacher-like disease 1, and related hypomyelinating disorders. Semin Neurol 32, 6267.

Ivanov, I. P., Atkins, J. F. and Michael, A. J. (2010). A profusion of upstream open reading frame mechanisms in polyamine-responsive translational regulation. Nucleic Acids Res 38, 353359.

Jackson, R. J., Hellen, C. U. and Pestova, T. V. (2010). The mechanism of eukaryotic translation initiation and principles of its regulation. Nat Rev Mol Cell Biol 11, 113-127.

Jazwinski, S. M. (1990). Preparation of extracts from yeast. Methods Enzymol 182, 154-174.

Jordanova, A., Irobi, J., Thomas, F. P., Van Dijck, P., Meerschaert, K., Dewil, M., Dierick, I., Jacobs, A., De Vriendt, E., Guergueltcheva, V., et al. (2006). Disrupted function and axonal distribution of mutant tyrosyl-tRNA synthetase in dominant intermediate Charcot-MarieTooth neuropathy. Nat Genet 38, 197-202.

Kodera, H., Osaka, H., Iai, M., Aida, N., Yamashita, A., Tsurusaki, Y., Nakashima, M., Miyake, N., Saitsu, H. and Matsumoto, N. (2015). Mutations in the glutaminyl-tRNA synthetase gene cause early-onset epileptic encephalopathy. J Hum Genet 60, 97-101.

Kozak, M. (1981). Possible role of flanking nucleotides in recognition of the AUG initiator codon by eukaryotic ribosomes. Nucleic Acids Res 9, 5233-5252.

Kozak, M. (1984). Compilation and analysis of sequences upstream from the translational start site in eukaryotic mRNAs. Nucleic Acids Res 12, 857-872.

Kozak, M. (1986). Point mutations define a sequence flanking the AUG initiator codon that modulates translation by eukaryotic ribosomes. Cell 44, 283-293.

Kozak, M. (1987). At least six nucleotides preceding the AUG initiator codon enhance translation in mammalian cells. J Mol Biol 196, 947-950.

Kozak, M. (1990). Downstream secondary structure facilitates recognition of initiator codons by eukaryotic ribosomes. Proc Natl Acad Sci U S A 87, 8301-8305. 
Kozak, M. (1997). Recognition of AUG and alternative initiator codons is augmented by G in position +4 but is not generally affected by the nucleotides in positions +5 and +6 . EMBO J 16, 2482-2492.

Kyriacou, S. V. and Deutscher, M. P. (2008). An important role for the multienzyme aminoacyltRNA synthetase complex in mammalian translation and cell growth. Mol Cell 29, 419427.

Latour, P., Thauvin-Robinet, C., Baudelet-Mery, C., Soichot, P., Cusin, V., Faivre, L., Locatelli, M. C., Mayencon, M., Sarcey, A., Broussolle, E., et al. (2010). A major determinant for binding and aminoacylation of tRNA ${ }^{\text {Ala }}$ in cytoplasmic alanyl-tRNA synthetase is mutated in dominant axonal Charcot-Marie-Tooth disease. Am J Hum Genet 86, 77-82.

Lei, H. Y., Zhou, X. L., Ruan, Z. R., Sun, W. C., Eriani, G. and Wang, E. D. (2015). Calpain cleaves most components in the multiple aminoacyl-tRNA synthetase complex and affects their functions. J Biol Chem 290, 26314-26327.

Li, J., Yao, Y. N., Liu, M. F. and Wang, E. D. (2003). Arginyl-tRNA synthetase with signature sequence KMSK from Bacillus stearothermophilus. Biochem J 376, 773-779.

Livak, K. J. and Schmittgen, T. D. (2001). Analysis of relative gene expression data using realtime quantitative PCR and the $2^{-\Delta \Delta \mathrm{C}_{\mathrm{T}}}$ method. Methods $25,402-408$.

Lossos, A., Elazar, N., Lerer, I., Schueler-Furman, O., Fellig, Y., Glick, B., Zimmerman, B. E., Azulay, H., Dotan, S., Goldberg, S., et al. (2015). Myelin-associated glycoprotein gene mutation causes Pelizaeus-Merzbacher disease-like disorder. Brain 138, 2521-2536.

Mehler, A. H. and Mitra, S. K. (1967). The activation of arginyl-transfer ribonucleic acid synthetase by transfer ribonucleic acid. J Biol Chem 242, 5495-5499.

Mendes, M. I., Green, L. M. C., Bertini, E., Tonduti, D., Aiello, C., Smith, D., Salsano, E., Beerepoot, S., Hertecant, J., von Spiczak, S., et al. (2020). RARSI-related hypomyelinating leukodystrophy: expanding the spectrum. Ann Clin Transl Neurol 7, 83-93.

Mudge, S. J., Williams, J. H., Eyre, H. J., Sutherland, G. R., Cowan, P. J. and Power, D. A. (1998). Complex organisation of the 5'-end of the human glycine tRNA synthetase gene. Gene 209, 45-50.

Nafisinia, M., Sobreira, N., Riley, L., Gold, W., Uhlenberg, B., Weiss, C., Boehm, C., Prelog, K., Ouvrier, R. and Christodoulou, J. (2017). Mutations in RARS cause a hypomyelination disorder akin to Pelizaeus-Merzbacher disease. Eur J Hum Genet 25, 1134-1141.

Negrutskii, B. S. and Deutscher, M. P. (1991). Channeling of aminoacyl-tRNA for protein synthesis in vivo. Proc Natl Acad Sci U S A 88, 4991-4995.

Nureki, O., Vassylyev, D. G., Tateno, M., Shimada, A., Nakama, T., Fukai, S., Konno, M., Hendrickson, T. L., Schimmel, P. and Yokoyama, S. (1998). Science 280, 578-582.

Papas, T. S. and Peterkofsky, A. (1972). A random sequential mechanism for arginyl-transfer ribonucleic acid synthetase of Escherichia coli. Biochemistry 11, 4602-4608. 
Raney, A., Law, G. L., Mize, G. J. and Morris, D. R. (2002). Regulated translation termination at the upstream open reading frame in S-adenosylmethionine decarboxylase mRNA. J Biol Chem 277, 5988-5994.

Rezaei, Z., Hosseinpour, S., Ashrafi, M. R., Mahdieh, N., Alizadeh, H., Mohammadpour, M., Khosroshahi, N., Amanat, M. and Tavasoli, A. R. (2019). Hypomyelinating leukodystrophy with spinal cord involvement caused by a novel variant in RARS: report of two unrelated patients. Neuropediatrics 50, 130-134.

Scheper, G. C., van der Klok, T., van Andel, R. J., van Berkel, C. G., Sissler, M., Smet, J., Muravina, T. I., Serkov, S. V., Uziel, G., Bugiani, M., et al. (2007). Mitochondrial aspartyltRNA synthetase deficiency causes leukoencephalopathy with brain stem and spinal cord involvement and lactate elevation. Nat Genet 39, 534-539.

Schwartzentruber, J., Buhas, D., Majewski, J., Sasarman, F., Papillon-Cavanagh, S., Thiffault, I., Sheldon, K. M., Massicotte, C., Patry, L., Simon, M., et al. (2014). Mutation in the nuclear-encoded mitochondrial isoleucyl-tRNA synthetase IARS2 in patients with cataracts, growth hormone deficiency with short stature, partial sensorineural deafness, and peripheral neuropathy or with Leigh syndrome. Hum Mutat 35, 1285-1289.

Sekine, S., Shimada, A., Nureki, O., Cavarelli, J., Moras, D., Vassylyev, D.G. and Yokoyama, S. (2001). Crucial role of the HIGH-loop lysine for the catalytic activity of arginyl-tRNA synthetase. J Biol Chem 276, 3723-3726.

Shiba, K., Schimmel, P., Motegi, H. and Noda, T. (1994). Human glycyl-tRNA synthetase: wide divergence of primary structure from bacterial counterpart and species-specific aminoacylation. J Biol Chem 269, 30049-30055.

Simon, M., Richard, E. M., Wang, X., Shahzad, M., Huang, V. H., Qaiser, T. A., Potluri, P., Mahl, S. E., Davila, A., Nazli, S., et al. (2015). Mutations of human NARS2, encoding the mitochondrial asparaginyl-tRNA synthetase, cause nonsyndromic deafness and Leigh syndrome. PLoS Genet 11, e1005097.

Sivaram, P. and Deutscher, M. P. (1990). Existence of two forms of rat liver arginyl-tRNA synthetase suggests channeling of aminoacyl-tRNA for protein synthesis. Proc Natl Acad Sci U S A 87, 3665-3669.

Steenweg, M. E., Ghezzi, D., Haack, T., Abbink, T. E., Martinelli, D., van Berkel, C. G., Bley, A., Diogo, L., Grillo, E., Te Water Naude, J., et al. (2012). Leukoencephalopathy with thalamus and brainstem involvement and high lactate 'LTBL' caused by EARS2 mutations. Brain 135, 1387-1394.

Studnicka, G. M. (1987). Hyperbolic regression analysis for kinetics, electrophoresis, ELISA, RIA, Bradford, Lowry, and other applications. Comput Appl Biosci 3, 9-16.

Taft, R. J., Vanderver, A., Leventer, R. J., Damiani, S. A., Simons, C., Grimmond, S. M., Miller, D., Schmidt, J., Lockhart, P. J., Pope, K., et al. (2013). Mutations in DARS cause 
hypomyelination with brain stem and spinal cord involvement and leg spasticity. Am J Hum Genet 92, 774-780.

Tolkunova, E., Park, H., Xia, J., King, M. P. and Davidson, E. (2000). The human lysyl-tRNA synthetase gene encodes both the cytoplasmic and mitochondrial enzymes by means of an unusual alternative splicing of the primary transcript. J Biol Chem 275, 35063-35069.

Varshavsky, A. (2011). The N-end rule pathway and regulation by proteolysis. Protein Sci 20, 1298-1345.

Vattem, K. M. and Wek, R. C. (2004). Reinitiation involving upstream ORFs regulates ATF4 mRNA translation in mammalian cells. Proc Natl Acad Sci U S A 101, 11269-11274.

Vellekamp, G. and Deutscher, M. P. (1987). A basic $\mathrm{NH}_{2}$-terminal extension of rat liver arginyltRNA synthetase required for its association with high molecular weight complexes. J Biol Chem 262, 9927-9930.

Wang, Y., Zhou, J. B., Zeng, Q. Y., Wu, S. Q., Xue, M. Q., Fang, P. F., Wang, E. D. and Zhou, X. L. (2020). Hearing impairment-associated KARS mutations lead to defects in aminoacylation of both cytoplasmic and mitochondrial tRNA ${ }^{\text {Lys }}$. Sci China Life Sci 63, 1227-1239.

Wei, J., Wu, C. and Sachs, M. S. (2012). The arginine attenuator peptide interferes with the ribosome peptidyl transferase center. Mol Cell Biol 32, 2396-2406.

Wei, N., Zhang, Q. and Yang, X. L. (2019). Neurodegenerative Charcot-Marie-Tooth disease as a case study to decipher novel functions of aminoacyl-tRNA synthetases. J Biol Chem 294, 5321-5339.

Werner, M., Feller, A., Messenguy, F. and Pierard, A. (1987). The leader peptide of yeast gene CPA1 is essential for the translational repression of its expression. Cell 49, 805-813.

Wolf, N. I., Salomons, G. S., Rodenburg, R. J., Pouwels, P. J., Schieving, J. H., Derks, T. G., Fock, J. M., Rump, P., van Beek, D. M., van der Knaap, M. S., et al. (2014). Mutations in RARS cause hypomyelination. Ann Neurol 76, 134-139.

Wu, J. F., Wang, E. D., Wang, Y. L., Gilbert, E. and Jean, G. (1999). Gene cloning, overproduction and purification of Escherichia coli $\mathrm{tRNA}^{\mathrm{Arg}}{ }_{2}$. Sheng Wu Hua Xue Yu Sheng Wu Wu Li Xue Bao (Shanghai) 31, 226-232.

Xu, W. L., Deng, B., Lin, P. H., Liu, C., Li, B., Huang, Q. J., Zhou, H., Yang, J. H. and Qu, L. H. (2020). Ribosome profiling analysis identified a KRAS-interacting microprotein that represses oncogenic signaling in hepatocellular carcinoma cells. Sci China Life Sci 63, 529-542.

Yang, F., Xia, X., Lei, H. Y. and Wang, E. D. (2010). Hemin binds to human cytoplasmic arginyl-tRNA synthetase and inhibits its catalytic activity. J Biol Chem 285, 39437-39446.

Young, S. K. and Wek, R. C. (2016). Upstream open reading frames differentially regulate genespecific translation in the integrated stress response. J Biol Chem 291, 16927-16935. 
Zheng, Y. G., Wei, H., Ling, C., Xu, M. G. and Wang, E. D. (2006). Two forms of human cytoplasmic arginyl-tRNA synthetase produced from two translation initiations by a single mRNA. Biochemistry 45, 1338-1344.

Zhou, X. L., Chen, Y., Zeng, Q. Y., Ruan, Z. R., Fang, P. F. and Wang, E. D. (2019). Newly acquired $\mathrm{N}$-terminal extension targets threonyl-tRNA synthetase-like protein into the multiple tRNA synthetase complex. Nucleic Acids Res 47, 8662-8674.

Zhou, X. L., He, L. X., Yu, L. J., Wang, Y., Wang, X. J., Wang, E. D. and Yang, T. (2017). Mutations in KARS cause early-onset hearing loss and leukoencephalopathy: potential pathogenic mechanism. Hum Mutat 38, 1740-1750.

Zhou, X. L., Ruan, Z. R., Huang, Q., Tan, M. and Wang, E. D. (2013). Translational fidelity maintenance preventing Ser mis-incorporation at Thr codon in protein from eukaryote. Nucleic Acids Res 41, 302-314.

Zhou, X. L. and Wang, E. D. (2013). Transfer RNA: A dancer between charging and mischarging for protein biosynthesis. Sci China Life Sci 56, 921-932.

Zhou, X. L., Zhu, B. and Wang, E. D. (2008). The CP2 domain of leucyl-tRNA synthetase is crucial for amino acid activation and post-transfer editing. J Biol Chem 283, 36608-36616. 


\section{FIGURES AND LEGENDS}

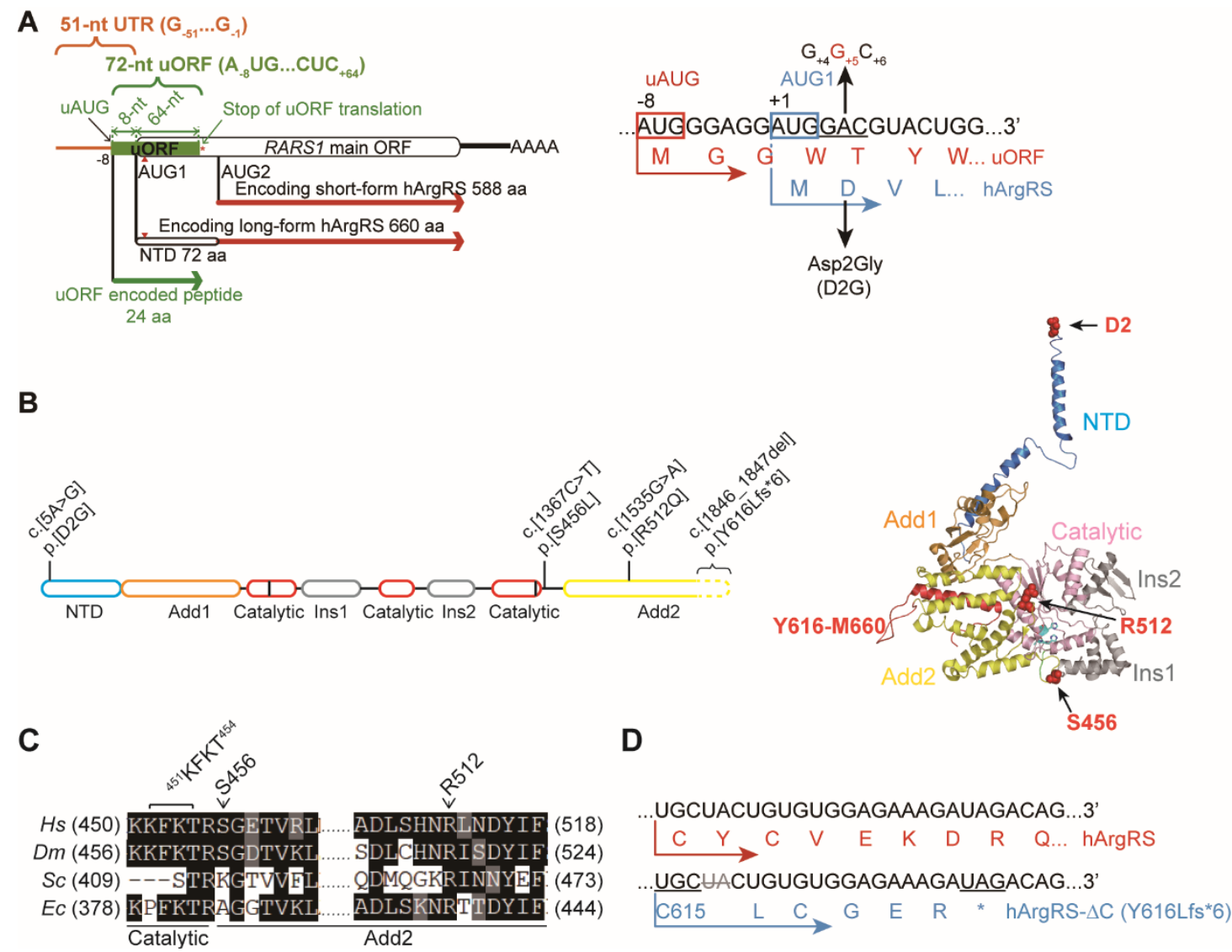

Figure 1. Locations of pathogenic mutations of hArgRS in its NTD and Add2 domain. (A)

Schematic representation (left panel) and partial sequence (right panel) of the 5'-mRNA of RARS1 gene. Translation from AUG1 and AUG2 in the main ORF produces the long and short form hArgRS (red arrows), respectively. A 5'-UTR (G-51 to $\mathrm{G}_{-1}$, orange line) contains an upstream AUG codon (uAUG) which initiates translation of an overlapping $\mathrm{uORF}$ (green box, $\mathrm{A}_{-8} \mathrm{U}_{-7} \mathrm{G}_{-6}$ to $\mathrm{U}_{+65} \mathrm{G}_{+66} \mathrm{~A}+67$ ) to produce a 24-aa peptide (green arrow). The c.[5A>G], p.[D2G] substitution is labelled by red triangle. In the partial sequence of 5'-mRNA, the UAUG and AUG1 are boxed and the partial peptide sequences encoded by two reading frames are shown with the c.[5A>G], p.[D2G] substitution labelled. (B) Distributions of the studied mutations on domain and structure of hArgRS. In hArgRS structure (PDB 4R3Z), the single-site mutation sites are shown as red spheres and the deleted peptide in Add2 domain caused by c.[1846_1847del] was labelled as red ribbon. (C) Partial multiple sequence alignment of a representative set of ArgRSs. S456 only exists in higher eukaryotes, and R512 is conserved in eukaryotic and prokaryotic ArgRSs. The "KMSK" peptide in aaRSs from E. coli to human is degenerate, and a

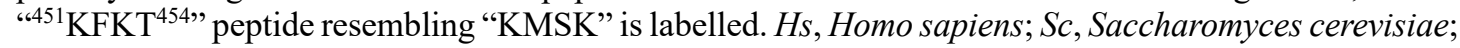
Ec, Escherichia coli; Gs, Geobacillus stearothermophilus. (D) The hArgRS- $\Delta \mathrm{C}$ variant is produced by a 2 -nt deletion. Translation is early-terminated at a rebuilt stop codon UAG. 


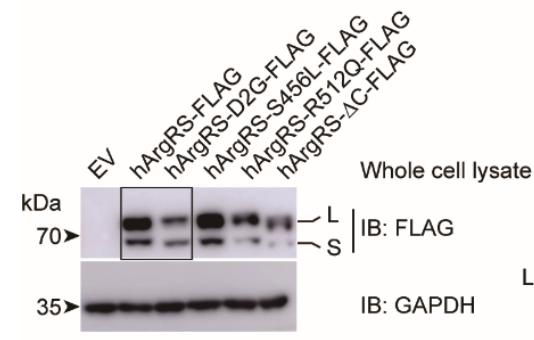

C

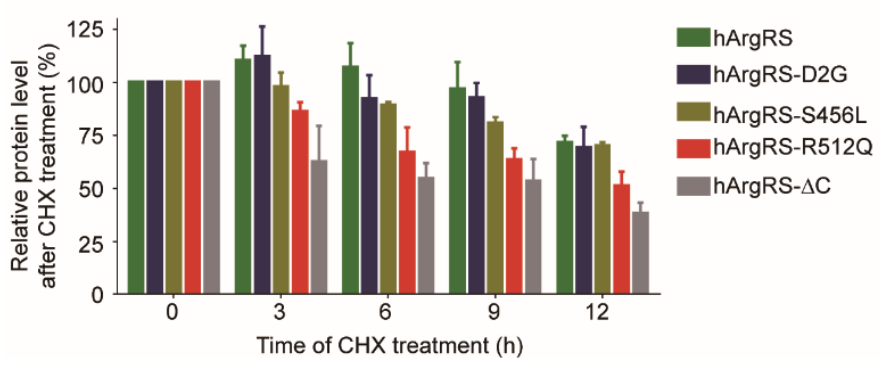

D
$+\mathrm{CHX}(\mathrm{h}) \quad \begin{array}{lllll}0 & 3 & 6 & 9 & 12\end{array}$

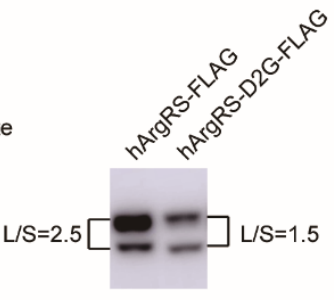

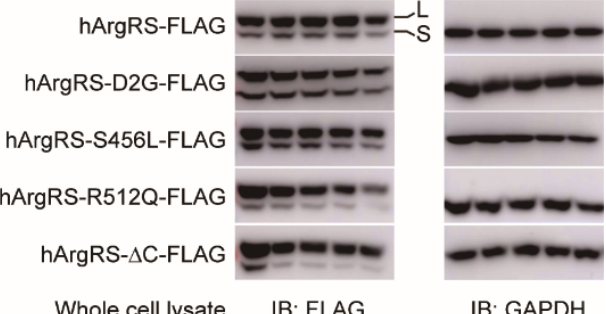

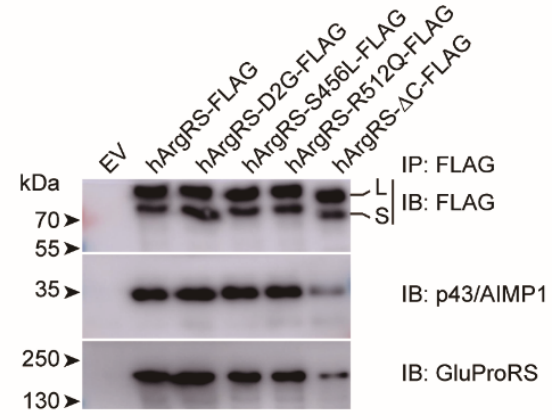

Figure 2. Pathogenic mutations in Add2 domain affect hArgRS stability and MSC assembly in cells. (A) Protein amounts of overexpressed hArgRS and its pathogenic variants in HEK293T cells. The amounts of hArgRS and hArgRS-D2G are shown in an inset with the ratio of long-form protein (indicated as "L") to short-form protein (indicated as "S") labelled. EV: empty vector control. (B and C) Protein amounts of hArgRS and its pathogenic variants after CHX treatment and their quantification. The longform and short-form hArgRS are indicated and labelled as "L" and "S", respectively. GAPDH was used as an internal reference in the CHX decay assay. The amount was quantified as the sum of long-form and short-form hArgRS, except for hArgRS-D2G, in which only the long-form hArgRS was quantified, as the short-form hArgRS does not contain the $\mathrm{D} 2 \mathrm{G}$ substitution. The results represent the mean $\pm \mathrm{SEM}$ from 3 independent experiments. (D) Co-IP using anti-FLAG antibody in HEK293T cells overexpressing FLAG-tagged hArgRS or its variants. The enriched MSC components (AIMP1 and hGluProRS) were detected by western blot. 
A
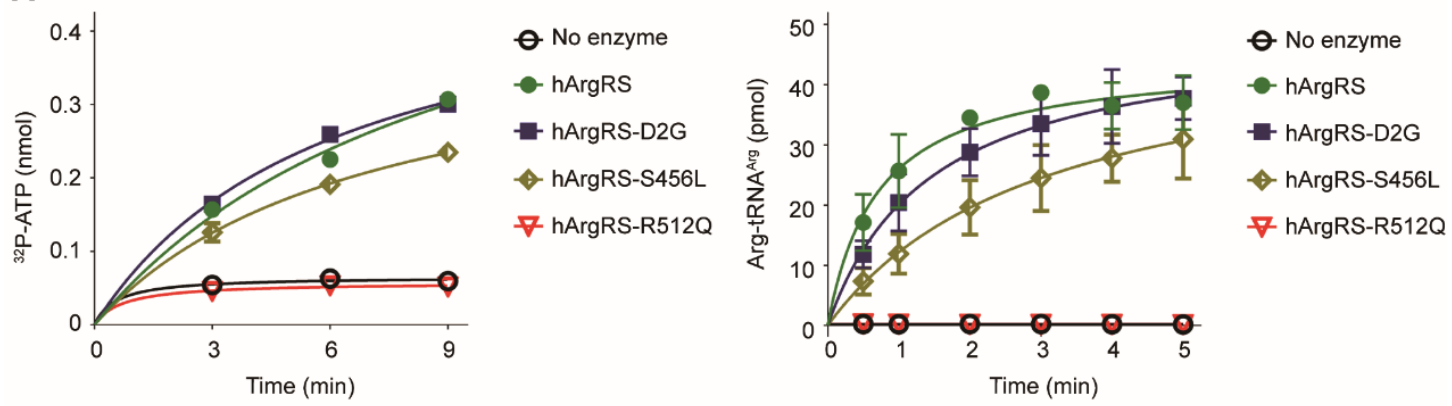

B
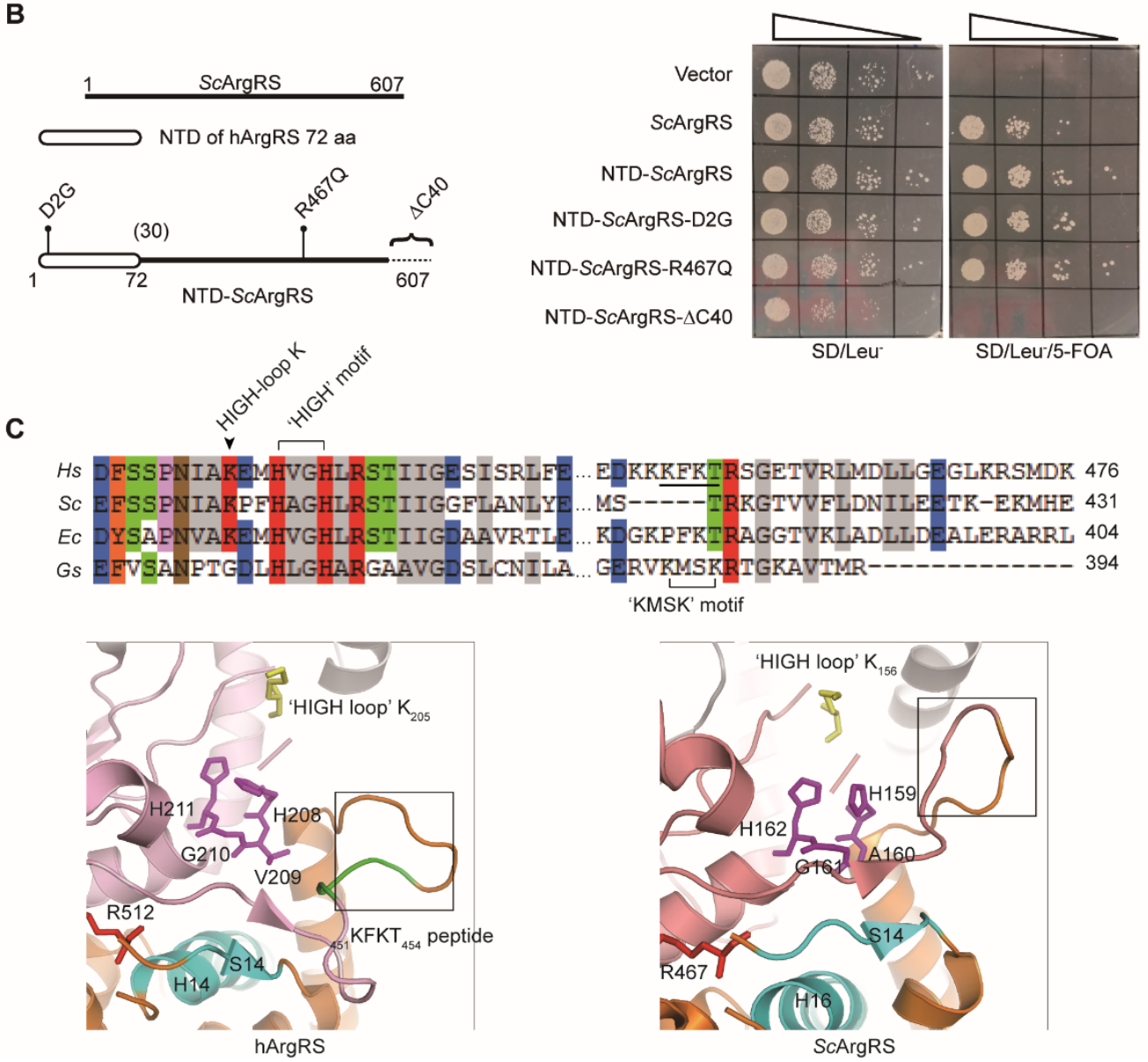

Figure 3. Catalytic activities of hArgRS pathogenic mutants and their ability to rescue the Sc $\triangle Y$ Y R341C yeast strain. (A) Arginine activation (left panel) and aminoacylation activities (right panel) of hArgRS and its pathogenic mutants. The results represent the mean \pm SEM from 3 independent experiments. (B) Construction and yeast complementation of NTD-ScArgRS or its variants. The mutated sites corresponding to hArgRS are shown. ScArgRS and empty vector were used as positive and negative controls, respectively. (C) Sequence alignment and structural representation of the active sites of hArgRS and ScArgRS. The conserved R512 in the hArgRS structure (PDB 4Q2T) and corresponding R467 in the ScArgRS structure (PDB 1F7V) in spatial proximity to the active site pocket are shown as red sticks. The conserved R residue lies between two helices in the Add2 domain (S14 and H14 in hArgRS and S14 and H16 in ScArgRS), which are colored in cyan. The "HIGH" motif and "HIGH"-loop K in the active site are shown as purple and yellow sticks, respectively. The "KMSK"-resembling " $451 \mathrm{KFKT}^{454 "}$ " peptide in $\mathrm{hArgRS}$ and the disordered peptide in this region of $S c \mathrm{ArgRS}$ are boxed. 
A
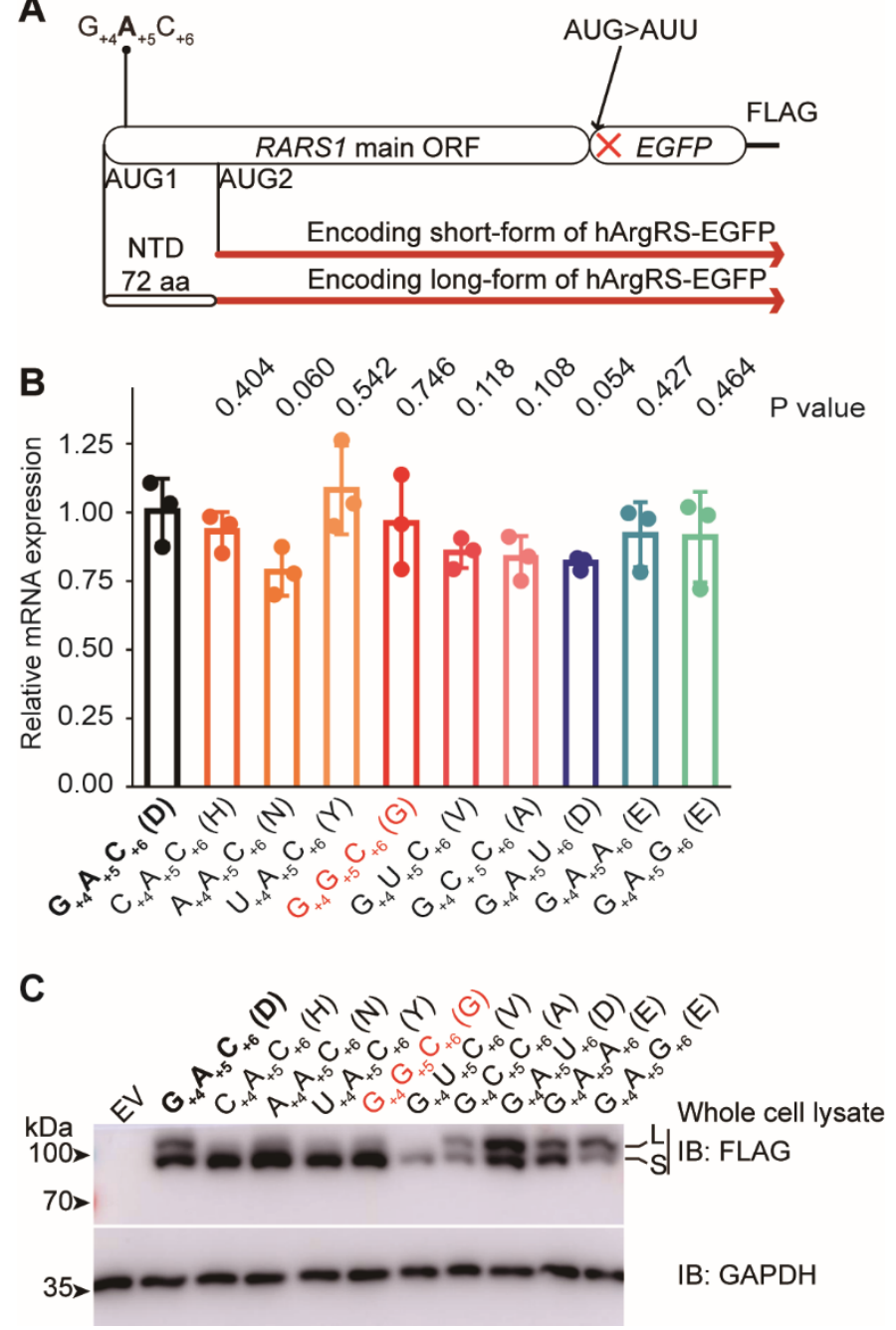

Figure 4. Nucleotides of codon 2 of hArgRS mRNA affect its translation in the absence of the 5'UTR. (A) Construction of the fused gene RARS1-EGFP-FLAG with codon 2 mutations in the absence of 5'-UTR. The red $\mathrm{x}$ indicates that AUG start codon of EGFP gene was mutated to AUU to prevent translation of free EGFP. The $\mathrm{G}_{+4} \mathrm{~A}+5 \mathrm{C}+6$ codon for $\mathrm{D} 2$ is labelled with the disease-causing substitution of $\mathrm{A}_{+5}$ shown in bold. (B) mRNA expression of RARS1-EGFP containing codon 2 substitutions in HEK293T cells by qPCR assay using primers specific for $E G F P$. ACTB was used for normalization. The native codon 2 and $\mathrm{D} 2$ amino acid is shown in bold, and the disease-causing mutation is colored in red. For all these constructs, the amino acids encoded by the mutated codons are shown in parentheses. The results represent the mean \pm SEM from 3 independent experiments. Each analysis group was compared with the native codon 2 construct, and the results were subjected to unpaired Student's t-test. (C) Effects of individual mutations of the $+4,+5$ and +6 nucleotides in codon 2 on the amount of hArgRS-EGFPFLAG and its mutants. All codon 2 mutants were overexpressed in HEK293T cells, and cell lysates were subjected to western blot using anti-FLAG antibody. Labels "L" and "S" indicate the long-form and short-form hArgRS-EGFP, respectively. 

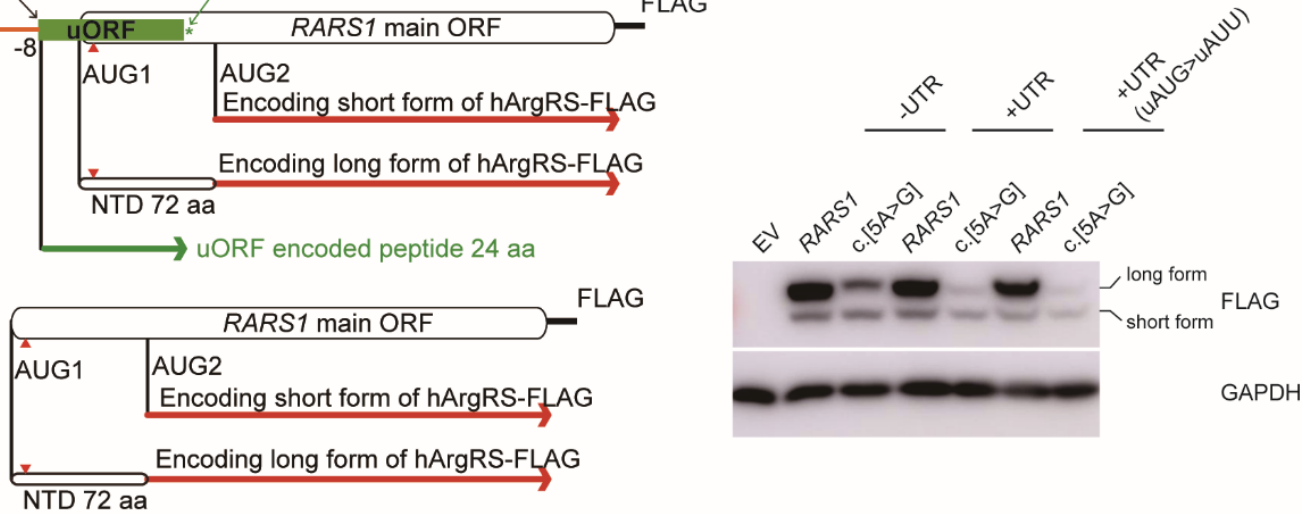

B

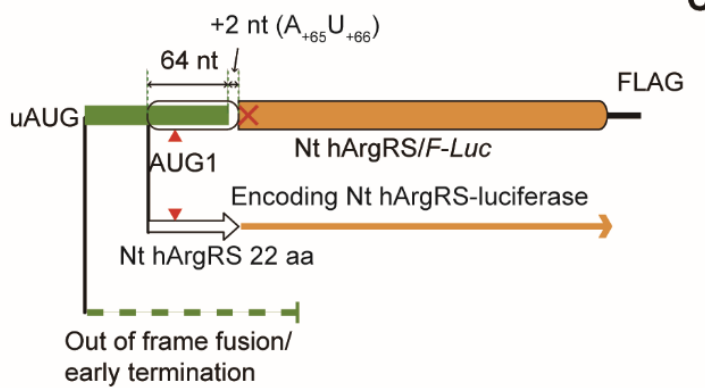

C

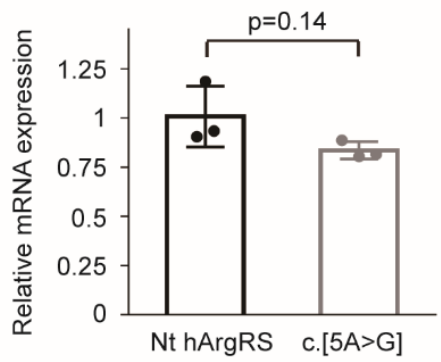

D
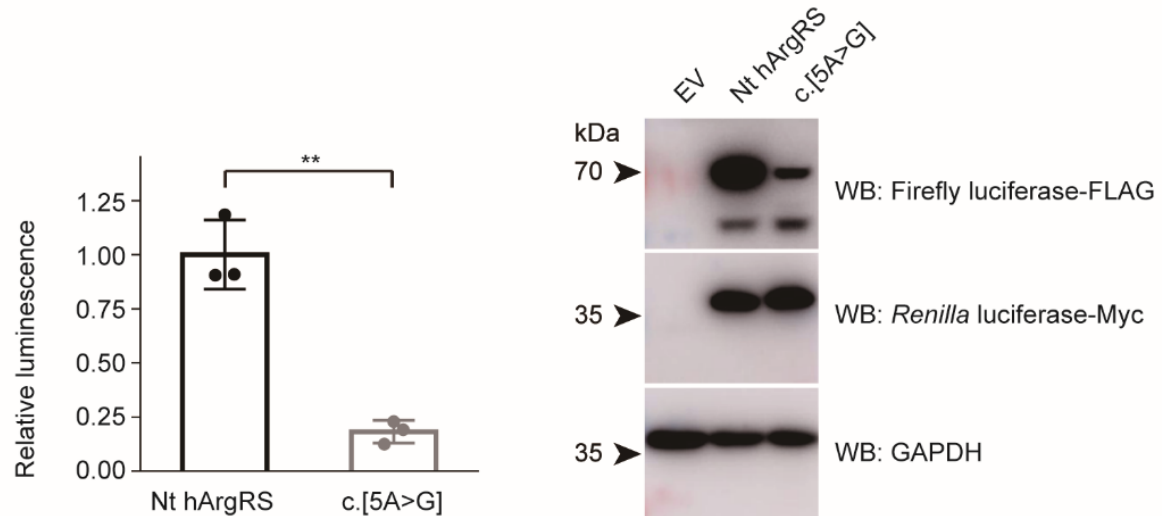

Figure 5. uORF mediates the inhibited translation of the downstream main ORF of mRNA containing the c. $[\mathbf{5 A}>\mathbf{G}]$ mutation. (A) Schematic representation (left panel) and protein amount (right panel) of the constructs with or without 5'-UTR tested for hArgRS synthesis. The marks used to indicate mRNA structural elements and pathogenic mutations are the same as in Figure 1A. Genes with a Cterminal FLAG-tag were overexpressed, and hArgRS produced from wild-type RARS1 gene as well as hArgRS-D2G produced produced from $R A R S 1$ gene containing c. $[5 \mathrm{~A}>\mathrm{G}]$ mutation were detected by western blot using anti-FLAG mAb (right panel). The two forms of hArgRS as well as their ratio were labelled. (B) Schematic representation of the construct Nt hArgRS/F-Luc for translation assay in the presence of the uORF. The coding sequence of Nt hArgRS (white bar) encoding a 22-aa peptide out of the NTD (white arrow) was fused with $F-L u c$ (with AUG start codon mutated to AUU) by inserting 2-nt at the junction of uORF (green box) and $F$-Luc coding sequence, while uORF is out of frame of $F$-Luc and its translation is early-terminated (green dashed line). (C) mRNA expression of Nt hArgRS/F-Luc or its mutant gene containing c.[5A>G] substitution in HEK293T cells by qPCR assay using primers specific for $F$-Luc gene. $A C T B$ was used for normalization. The results represent the mean \pm SEM from 3 independent experiments. Expression of the mutant gene was compared with the native construct, and the result was subjected to unpaired Student's t-test. (D) Production of Nt hArgRS-luciferase or its mutant from constructs shown in Figure 5B. For the dual-luciferase assay, relative luminescence was represented as the ratio of firefly luciferase activity to Renilla luciferase activity (left panel). In western blot, co-expressed Renilla luciferase with myc-tag was used as a standard control (right panel). The results represent the mean \pm SEM from 3 independent experiments. Expression of the mutant gene was compared with the native construct, and the result was subjected to unpaired Student's t-test. 
Table 1. PMLD-related ${ }^{\mathrm{a}} \mathrm{R} A R S 1$ mutations and their frequencies

\begin{tabular}{lll}
\hline cDNA & Protein & ${ }^{\mathrm{b}}$ Frequencies \\
\hline$[5 \mathrm{~A}>\mathrm{G}]$ & {$[\mathrm{D} 2 \mathrm{G}]$} & $10 / 25$ \\
{$[1535 \mathrm{G}>\mathrm{G}]$} & {$[\mathrm{R} 512 \mathrm{Q}]$} & $2 / 25$ \\
{$[1367 \mathrm{C}>\mathrm{T}]$} & {$[\mathrm{S} 456 \mathrm{~L}]$} & $2 / 25$ \\
{$\left[1846 \_1847 \mathrm{del}\right]$} & {$[$ Y $616 \mathrm{Lfs} * 6]$} & $1 / 25$ \\
\hline
\end{tabular}

${ }^{\mathrm{a}}$ RefSeq NG_041809.1

${ }^{\mathrm{b}}$ Frequencies represent the number of patients out of all 25 RARS1 mutation-related PMLD

cases 
Table 2. Proportions of secondary structures and melting temperatures of ArgRS and its pathogenic mutants in solution

\begin{tabular}{lccccc}
\hline & ${ }^{\mathrm{a}}$ Helix & ${ }^{\mathrm{a}}$ Sheet & ${ }^{\mathrm{a}}$ Turn & ${ }^{\mathrm{a}}$ Random coil & ${ }^{\mathrm{b}} \mathrm{T}_{\mathrm{m}}$ \\
\hline hArgRS & 43.76 & 21.53 & 6.81 & 27.90 & $44.8 \pm 0.17$ \\
hArgRS-D2G & 44.28 & 21.17 & 6.90 & 27.65 & $45.1 \pm 0.08$ \\
hArgRS-S456L & 43.85 & 21.23 & 7.82 & 27.10 & $44.5 \pm 0.27$ \\
hArgRS-R512Q & 48.35 & 11.09 & 17.73 & 22.83 & $46.5 \pm 0.12$ \\
\hline
\end{tabular}

${ }^{a}$ Fractions of secondary structures were calculated by comparing molar residue ellipticity with reference structures over two accumulative scans.

${ }^{\mathrm{b}}$ Data represent the mean $\pm \mathrm{SEM}$ from three independent experiments. 
Table 3. Kinetic parameters of hArgRS and its pathogenic mutants for tRNA ${ }^{\mathrm{Arg}}$ in aminoacylation reaction

\begin{tabular}{lllll}
\hline & ${ }^{\mathrm{a}} k_{\text {cat }}\left(\mathrm{s}^{-1}\right)$ & ${ }^{\mathrm{a}} K_{m}(\mu \mathrm{M})$ & $k_{\mathrm{cat}} / K_{m}\left(\mu \mathrm{M}^{-1} \cdot \mathrm{s}^{-1}\right)$ & ${ }^{\mathrm{c}}$ Relative $k_{\text {cat }} / K_{m}(\%)$ \\
\hline hArgRS & $9.99 \pm 0.54$ & $8.55 \pm 0.78$ & 1.17 & 100 \\
hArgRS-D2G & $8.78 \pm 0.86$ & $7.68 \pm 0.74$ & 1.14 & 97.4 \\
hArgRS-S456L & $1.73 \pm 0.08$ & $2.82 \pm 0.11$ & 0.61 & 52.1 \\
hArgRS-R512Q & ${ }^{\mathrm{b}} \mathrm{ND}$ & ${ }^{\mathrm{b}} \mathrm{ND}$ & ${ }^{\mathrm{b}} \mathrm{ND}$ & ${ }^{\mathrm{b}} \mathrm{ND}$ \\
\hline
\end{tabular}

${ }^{\mathrm{a}}$ Data represent the mean $\pm \mathrm{SEM}$ from three independent experiments.

${ }^{\mathrm{b}} \mathrm{ND}$, not determined; too low to be determined.

${ }^{\mathrm{c}}$ The relative catalytic efficiency of hArgRS was $100 \%$. 